\title{
Chiral Norbornadienes as Efficient Ligands for the Rhodium-Catalyzed Asymmetric 1,4-Addition of Arylboronic Acids to Fumaric and Maleic Compouds
}

Ryo Shintani, Kazuhito Ueyama, Ichiro Yamada, and Tamio Hayashi*

Department of Chemistry, Graduate School of Science, Kyoto University, Sakyo, Kyoto 606-8502, Japan

\section{Supporting Information}

\section{General}

All manipulations were carried out with standard Schlenk techniques under nitrogen.

1,4-Dioxane and $\mathrm{Et}_{2} \mathrm{O}$ were distilled over benzophenone ketyl under nitrogen.

$N$-methylmaleimide (Aldrich), N-benzylmaleimide (ICN Biomedicals), phenylboronic acid (TCI), and $\mathrm{LiAlH}_{4}$ (Wako Chemicals) were used as received. $\left[\mathrm{RhCl}\left(\mathrm{C}_{2} \mathrm{H}_{4}\right)_{2}\right]_{2}{ }^{1}$ and $(1 R, 4 R)$-2,5-dibenzylbicyclo[2.2.1]hepta-2,5-diene $(5 \mathbf{a})^{2}$ were prepared following the literature procedures. $(1 R, 4 R)-2,5$-bis $(2,4,6-$ trimethylphenylmethyl)bicyclo[2.2.1]hepta-2,5-diene (5b) was synthesized following the procedure for $(R, R)-5 \mathbf{a}$. $N$-cyclohexylmaleimide ${ }^{3}$ and di-tert-butyl fumarate ${ }^{4}$ were $^{-}$ synthesized following the literature procedures.

All other chemicals and solvents were purchased from Aldrich, Wako Chemicals, TCI, or Kanto Chemicals and used as received.

\section{Spectra Data for $(R, R)-5 b$ and Its Synthetic Intermediates}<smiles>Cc1cc(C)c(CC2=CC3CC2CC32OCCO2)c(C)c1</smiles>

(1R,4R)-Spiro[5-(2,4,6-trimethylphenylmethyl)bicyclo[2.2.1]hepta-5-ene-2,2'-

\section{$[1,3]$ dioxolane $]$}

${ }^{1} \mathrm{H} \mathrm{NMR}\left(\mathrm{CDCl}_{3}\right): \delta 6.82(\mathrm{~s}, 2 \mathrm{H}), 5.24\left(\mathrm{~d},{ }^{3} \mathrm{~J}_{\mathrm{HH}}=1.6 \mathrm{~Hz}, 1 \mathrm{H}\right), 3.98-3.77(\mathrm{~m}, 4 \mathrm{H}), 3.44$ $\left(\mathrm{dd},{ }^{2} J_{\mathrm{HH}}=17.1 \mathrm{~Hz}\right.$ and $\left.{ }^{4} J_{\mathrm{HH}}=1.8 \mathrm{~Hz}, 1 \mathrm{H}\right), 3.31\left(\mathrm{dd},{ }^{2} J_{\mathrm{HH}}=17.1 \mathrm{~Hz}\right.$ and ${ }^{4} J_{\mathrm{HH}}=1.8 \mathrm{~Hz}$, $1 \mathrm{H})$, 2.59-2.55 (m, 1H), 2.54-2.52 (m, 1H), $2.25(\mathrm{~s}, 3 \mathrm{H}), 2.20(\mathrm{~s}, 6 \mathrm{H}), 1.87\left(\mathrm{dd},{ }^{2} J_{\mathrm{HH}}=12.3\right.$ $\mathrm{Hz}$ and $\left.{ }^{3} J_{\mathrm{HH}}=3.8 \mathrm{~Hz}, 1 \mathrm{H}\right), 1.70\left(\mathrm{~d},{ }^{2} J_{\mathrm{HH}}=8.6 \mathrm{~Hz}, 1 \mathrm{H}\right), 1.64\left(\mathrm{ddt},{ }^{2} J_{\mathrm{HH}}=8.6 \mathrm{~Hz},{ }^{3} J_{\mathrm{HH}}=3.5\right.$ $\mathrm{Hz}$, and $\left.{ }^{4} J_{\mathrm{HH}}=1.8 \mathrm{~Hz}, 1 \mathrm{H}\right), 1.46\left(\mathrm{dd},{ }^{2} J_{\mathrm{HH}}=12.3 \mathrm{~Hz}\right.$ and $\left.{ }^{3} J_{\mathrm{HH}}=3.5 \mathrm{~Hz}, 1 \mathrm{H}\right) .{ }^{13} \mathrm{C} \mathrm{NMR}$ $\left(\mathrm{CDCl}_{3}\right): \delta 151.0,136.7,135.2,132.6,128.7,125.3,119.4,64.7,64.3,49.6,48.3,44.1,39.8$, 30.3, 20.9, 19.7. Anal. Calcd for $\mathrm{C}_{19} \mathrm{H}_{24} \mathrm{O}_{2}$ : C, 80.24; $\mathrm{H}, 8.51$. Found: $\mathrm{C}, 80.41 ; \mathrm{H}, 8.79$.

\footnotetext{
${ }^{1}$ Cramer, R. Inorg. Synth. 1974, 15, 16.

${ }^{2}$ Hayashi, T.; Ueyama, K.; Tokunaga, N.; Yoshida, K. J. Am. Chem. Soc. 2003, 125, 11508.

${ }^{3}$ Kita, Y.; Tobo, T.; Sakamoto, K.; Baba, M.; Nakagawa, Y. Jpn. Kokai Tokkyo Koho 1988, 7.

${ }^{4}$ Tsuge, O.; Kanemasa, S.; Takenaka, S. Bull. Chem. Soc., Jpn. 1985, 58, 3137.
} 


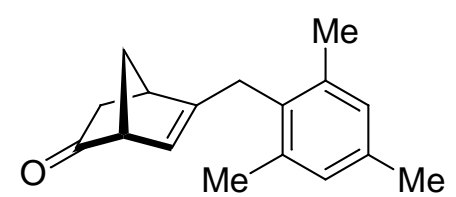

(1R,4R)-2-(2,4,6-Trimethylphenylmethyl)[2.2.1]hepta-2-en-5-one

${ }^{1} \mathrm{H} \mathrm{NMR}\left(\mathrm{CDCl}_{3}\right): \delta 6.82(\mathrm{~s}, 2 \mathrm{H}), 5.23-5.20(\mathrm{~m}, 1 \mathrm{H}), 3.52\left(\mathrm{dd},{ }^{2} J_{\mathrm{HH}}=17.6 \mathrm{~Hz}\right.$ and ${ }^{4} J_{\mathrm{HH}}$ $=2.0 \mathrm{~Hz}, 1 \mathrm{H}), 3.35\left(\mathrm{dd},{ }^{2} J_{\mathrm{HH}}=17.6 \mathrm{~Hz}\right.$ and $\left.{ }^{4} J_{\mathrm{HH}}=2.0 \mathrm{~Hz}, 1 \mathrm{H}\right), 2.92-2.89(\mathrm{~m}, 2 \mathrm{H}), 2.25(\mathrm{~s}$, $3 \mathrm{H}), 2.18(\mathrm{~s}, 6 \mathrm{H}), 2.13\left(\mathrm{ddt},{ }^{2} J_{\mathrm{HH}}=11.0 \mathrm{~Hz},{ }^{3} J_{\mathrm{HH}}=4.4 \mathrm{~Hz}\right.$, and $\left.{ }^{4} J_{\mathrm{HH}}=2.4 \mathrm{~Hz}, 1 \mathrm{H}\right), 1.98$ $\left(\mathrm{dd},{ }^{2} J_{\mathrm{HH}}=16.5 \mathrm{~Hz}\right.$ and $\left.{ }^{3} J_{\mathrm{HH}}=3.7 \mathrm{~Hz}, 1 \mathrm{H}\right), 1.92\left(\mathrm{~d},{ }^{2} J_{\mathrm{HH}}=11.0 \mathrm{~Hz}, 1 \mathrm{H}\right), 1.78\left(\mathrm{dd},{ }^{2} J_{\mathrm{HH}}=\right.$ $16.5 \mathrm{~Hz}$ and $\left.{ }^{3} \mathrm{~J}_{\mathrm{HH}}=4.4 \mathrm{~Hz}, 1 \mathrm{H}\right) .{ }^{13} \mathrm{C} \mathrm{NMR}\left(\mathrm{CDCl}_{3}\right): \delta 214.5,155.7,136.4,135.7,131.3$, 128.8, 122.0, 55.9, 49.6, 43.2, 37.0, 30.8, 20.8, 19.7. Anal. Calcd for $\mathrm{C}_{17} \mathrm{H}_{20} \mathrm{O}: \mathrm{C}, 84.96$; $\mathrm{H}$, 8.39. Found: $\mathrm{C}, 85.11 ; \mathrm{H}, 8.39$.

(1R,4R)-2-(2,4,6-trimethyphenylmethyl)-5-

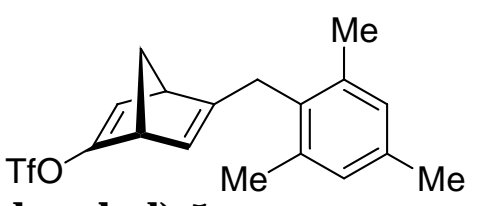

\section{trifluoromethanesulfonyloxybicyclo[2.2.1]hepta-2,5-diene}

${ }^{1} \mathrm{H} \mathrm{NMR}\left(\mathrm{CDCl}_{3}\right): \delta 6.82(\mathrm{~s}, 2 \mathrm{H}), 6.30-6.27(\mathrm{~m}, 1 \mathrm{H}), 5.95-5.92(\mathrm{~m}, 1 \mathrm{H}), 3.53\left(\mathrm{~d},{ }^{2} J_{\mathrm{HH}}=\right.$ $17.6 \mathrm{~Hz}, 1 \mathrm{H}), 3.40\left(\mathrm{~d},{ }^{2} J_{\mathrm{HH}}=17.6 \mathrm{~Hz}, 1 \mathrm{H}\right), 3.33-3.28(\mathrm{~m}, 2 \mathrm{H}), 2.37-2.34(\mathrm{~m}, 1 \mathrm{H}), 2.25(\mathrm{~s}$, $3 \mathrm{H}), 2.20-2.17(\mathrm{~m}, 1 \mathrm{H}), 2.16(\mathrm{~s}, 6 \mathrm{H}) .{ }^{13} \mathrm{C} \mathrm{NMR}\left(\mathrm{CDCl}_{3}\right): \delta 168.2,157.7,136.4,135.5,132.2$, 132.1, 128.8, 124.2, $118.6\left(\mathrm{q},{ }^{1} J_{\mathrm{CF}}=320 \mathrm{~Hz}\right), 72.1,51.9,51.4,31.3,20.9,19.6$. Anal. Calcd for $\mathrm{C}_{18} \mathrm{H}_{19} \mathrm{~F}_{3} \mathrm{O}_{3} \mathrm{~S}: \mathrm{C}, 58.05 ; \mathrm{H}, 5.14$. Found: $\mathrm{C}, 58.00 ; \mathrm{H}, 5.15$.

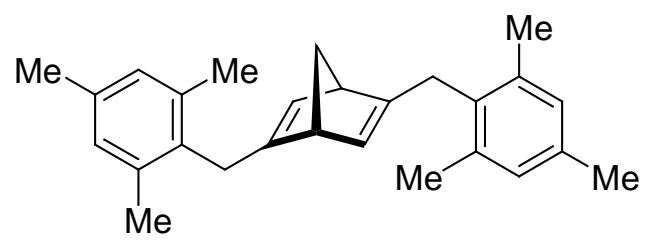

(1R,4R)-2,5-Di(2,4,6-trimethylphenylmethyl)bicyclo[2.2.1]hepta-2,5-diene (5b)

${ }^{1} \mathrm{H} \mathrm{NMR}\left(\mathrm{CDCl}_{3}\right): \delta 6.80(\mathrm{~s}, 4 \mathrm{H}), 5.72\left(\mathrm{dt},{ }^{3} J_{\mathrm{HH}}=3.9 \mathrm{~Hz}\right.$ and $\left.{ }^{4} J_{\mathrm{HH}}=1.9 \mathrm{~Hz}, 2 \mathrm{H}\right), 3.46$ $\left(\mathrm{dd},{ }^{2} J_{\mathrm{HH}}=17.4 \mathrm{~Hz}\right.$ and $\left.{ }^{4} J_{\mathrm{HH}}=1.9 \mathrm{~Hz}, 2 \mathrm{H}\right), 3.27\left(\mathrm{dd},{ }^{2} J_{\mathrm{HH}}=17.4 \mathrm{~Hz}\right.$ and ${ }^{4} J_{\mathrm{HH}}=1.9 \mathrm{~Hz}$, $2 \mathrm{H}), 3.15-3.12(\mathrm{~m}, 2 \mathrm{H}), 2.25(\mathrm{~s}, 6 \mathrm{H}), 2.14(\mathrm{~s}, 12 \mathrm{H}), 1.98-1.96(\mathrm{~m}, 2 \mathrm{H}) .{ }^{13} \mathrm{C} \mathrm{NMR}\left(\mathrm{CDCl}_{3}\right): \delta$ $157.1,136.4,135.0,133.5,132.5,128.5,71.5,53.5,31.6,20.9,19.7 . \alpha]^{20}-77.3$ (c 1.00, $\mathrm{CHCl}_{3}$ ). Anal. Calcd for $\mathrm{C}_{27} \mathrm{H}_{32}$ : C, 90.95; H, 9.05. Found: $\mathrm{C}, 91.95 ; \mathrm{H}, 9.16$.

\section{1,4-Addition Reactions}

\section{General Procedure for Table 1.}

A solution of $\left[\mathrm{RhCl}\left(\mathrm{C}_{2} \mathrm{H}_{4}\right)_{2}\right]_{2}(1.9 \mathrm{mg}, 9.8 \mu \mathrm{mol} \mathrm{Rh})$ and chiral ligand $(11 \mu \mathrm{mol})$ in 1,4-dioxane $(1.0 \mathrm{~mL})$ was stirred for $10 \mathrm{~min}$ at room temperature. $\mathrm{KOH}(0.20 \mathrm{~mL}, 20$ $\mu$ mol; $0.1 \mathrm{M}$ aqueous) was then added to it, and the resulting solution was stirred for additional $10 \mathrm{~min}$ at room temperature. After addition of $\mathrm{ArB}(\mathrm{OH})_{2}(0.60 \mathrm{mmol})$ and stirring for $5 \mathrm{~min}$, this mixture was transferred to a solution of di-tert-butyl fumarate $(45.6 \mathrm{mg}, 0.20 \mathrm{mmol})$ in 1,4-dioxane $(1.0 \mathrm{~mL})$. The resulting mixture was stirred for $3 \mathrm{~h}$ 
at $50{ }^{\circ} \mathrm{C}$, and was then passed through a pad of silica gel with $\mathrm{Et}_{2} \mathrm{O}$, and the solvent was removed under vacuum. The residue was purified by silica gel PTLC with EtOAc/hexane afford the desired 1,4-adduct 6.<smiles>CCCCOC(=O)C[C@@H](C(=O)OCCC)c1ccccc1</smiles>

Table 1, entry 6 (6a). (CAS 159528-25-3) Pale yellow oil. 90\% yield. The ee was determined on a Daicel Chiralpak AD-H column with hexane : isopropanol = $95: 5$, flow $=1.0 \mathrm{~mL} / \mathrm{min}$. Retention times: $8.1 \mathrm{~min}[(R)$-enantiomer $], 16.6 \mathrm{~min}[(S)-$ enantiomer]. 90\% ee. $\left.\_\right]^{20}{ }_{\mathrm{D}}+57.4\left(\mathrm{c} 0.99, \mathrm{CHCl}_{3}\right)$.

${ }^{1} \mathrm{H}$ NMR $\left(\mathrm{CDCl}_{3}\right): \delta 7.75-7.23(\mathrm{~m}, 5 \mathrm{H}), 3.92\left(\mathrm{dd},{ }^{3} \mathrm{~J}_{\mathrm{HH}}=10.0 \mathrm{~Hz}\right.$ and $\left.5.7 \mathrm{~Hz}, 1 \mathrm{H}\right), 3.01$ $\left(\mathrm{dd},{ }^{2} J_{\mathrm{HH}}=16.5 \mathrm{~Hz}\right.$ and $\left.{ }^{3} J_{\mathrm{HH}}=10.0 \mathrm{~Hz}, 1 \mathrm{H}\right), 2.55\left(\mathrm{dd},{ }^{2} J_{\mathrm{HH}}=16.5 \mathrm{~Hz}\right.$ and ${ }^{3} J_{\mathrm{HH}}=5.7 \mathrm{~Hz}$, $1 \mathrm{H}), 1.40(\mathrm{~s}, 9 \mathrm{H}), 1.39(\mathrm{~s}, 9 \mathrm{H})$.

The absolute configuration was determined by comparison of the optical rotation with that reported in the literature, ${ }^{5}$ after derivatization to 2-phenylbutane-1,4-diol 7 (CAS 6837-05-4) following the procedure below:

$\mathrm{LiAlH}_{4}(7.6 \mathrm{mg}, 0.20 \mathrm{mmol})$ was added to a solution of the 1,4-adduct $(30.9 \mathrm{mg}, 0.10$ $\mathrm{mmol})$ in $\mathrm{Et}_{2} \mathrm{O}(1.0 \mathrm{~mL})$, and the mixture was stirred for $10 \mathrm{~h}$ at room temperature. After quenching with a drop of water, this was passed through a pad of silica gel with $\mathrm{Et}_{2} \mathrm{O}$ to afford the desired diol as a colorless oil $(8.4 \mathrm{mg}, 0.51 \mathrm{mmol} ; 51 \%$ yield $\left.) . \_\alpha\right]^{20}$ $+28.2(c 0.84, \mathrm{MeOH})$.

${ }^{1} \mathrm{H}$ NMR $\left(\mathrm{CDCl}_{3}\right): \delta 7.32\left(\mathrm{t},{ }^{3} \mathrm{~J}_{\mathrm{HH}}=7.4 \mathrm{~Hz}, 2 \mathrm{H}\right), 7.27-7.19(\mathrm{~m}, 3 \mathrm{H}), 3.77\left(\mathrm{~d},{ }^{3} \mathrm{~J}_{\mathrm{HH}}=6.8\right.$ $\mathrm{Hz}, 2 \mathrm{H}), 3.68\left(\mathrm{dt},{ }^{2} J_{\mathrm{HH}}=10.8 \mathrm{~Hz}\right.$ and $\left.{ }^{3} J_{\mathrm{HH}}=5.6 \mathrm{~Hz}, 1 \mathrm{H}\right), 3.57\left(\mathrm{ddd},{ }^{2} J_{\mathrm{HH}}=10.8 \mathrm{~Hz}\right.$ and ${ }^{3} J_{\mathrm{HH}}=8.1 \mathrm{~Hz}$ and $\left.5.6 \mathrm{~Hz}, 1 \mathrm{H}\right), 2.95\left(\right.$ quint, $\left.{ }^{3} J_{\mathrm{HH}}=6.8 \mathrm{~Hz}, 1 \mathrm{H}\right), 2.24(\mathrm{bs}, 2 \mathrm{H}), 2.02(\mathrm{ddt}$, ${ }^{2} J_{\mathrm{HH}}=13.8 \mathrm{~Hz}$ and ${ }^{3} J_{\mathrm{HH}}=12.2 \mathrm{~Hz}$ and $\left.5.6 \mathrm{~Hz}, 1 \mathrm{H}\right), 1.89\left(\mathrm{ddt},{ }^{2} J_{\mathrm{HH}}=13.8 \mathrm{~Hz}\right.$ and ${ }^{3} \mathrm{~J}_{\mathrm{HH}}=$ $8.1 \mathrm{~Hz}$ and $5.6 \mathrm{~Hz}, 1 \mathrm{H})$.<smiles>CCCCOC(=O)C[C@H](C(=O)OCCC)c1ccc(OC)cc1</smiles>

Table 1, entry 7 (6b). Pale yellow oil. $78 \%$ yield. The ee was determined on a Daicel Chiralpak AD-H column with hexane : isopropanol $=95: 5$, flow $=1.0 \mathrm{~mL} / \mathrm{min}$. Retention times: 13.8 min [(R)-enantiomer], 30.1 min [(S)-enantiomer]. $86 \%$ ee. $\left.\_\alpha\right]^{20}$ $+55.7\left(c\right.$ 1.31, $\left.\mathrm{CHCl}_{3}\right)$. The absolute configuration was assigned by analogy with Table 2 , entry 1.

${ }^{1} \mathrm{H} \mathrm{NMR}\left(\mathrm{CDCl}_{3}\right): \delta 7.19\left(\mathrm{~d},{ }^{3} J_{\mathrm{HH}}=8.7 \mathrm{~Hz}, 2 \mathrm{H}\right), 6.84\left(\mathrm{~d},{ }^{3} J_{\mathrm{HH}}=8.7 \mathrm{~Hz}, 2 \mathrm{H}\right), 3.86(\mathrm{dd}$, ${ }^{3} J_{\mathrm{HH}}=10.0 \mathrm{~Hz}$ and $\left.5.8 \mathrm{~Hz}, 1 \mathrm{H}\right), 3.78(\mathrm{~s}, 3 \mathrm{H}), 2.98\left(\mathrm{dd},{ }^{2} J_{\mathrm{HH}}=16.4 \mathrm{~Hz}\right.$ and ${ }^{3} J_{\mathrm{HH}}=10.0 \mathrm{~Hz}$, $1 \mathrm{H}), 2.52\left(\mathrm{dd},{ }^{2} \mathrm{JHH}_{\mathrm{HH}}=16.4 \mathrm{~Hz}\right.$ and $\left.{ }^{3} \mathrm{~J}_{\mathrm{HH}}=5.8 \mathrm{~Hz}, 1 \mathrm{H}\right), 1.40(\mathrm{~s}, 9 \mathrm{H}), 1.38(\mathrm{~s}, 9 \mathrm{H}) .{ }^{13} \mathrm{C} \mathrm{NMR}$ $\left(\mathrm{CDCl}_{3}\right): \delta 172.3,170.9,158.7,130.6,128.7,114.0,80.8,80.6,55.2,47.6,39.2,28.0,27.9$. Anal. Calcd for $\mathrm{C}_{19} \mathrm{H}_{28} \mathrm{O}_{5}: \mathrm{C}, 67.83 ; \mathrm{H}, 8.39$. Found: $\mathrm{C}, 67.64 ; \mathrm{H}, 8.32$.

\footnotetext{
${ }^{5}$ Agami, C.; Couty, F.; Evano, G. Eur. J. Org. Chem. 2002, 29.
} 
<smiles>CCCCOC(=O)C[C@H](C(=O)OCCC)c1ccc(F)cc1</smiles>

Table 1, entry 8 (6c). Pale yellow oil. 85\% yield. The ee was determined on a Daicel Chiralpak AD-H column with hexane : isopropanol $=95: 5$, flow $=1.0 \mathrm{~mL} / \mathrm{min}$. Retention times: $11.0 \mathrm{~min}\left[(R)\right.$-enantiomer], $24.9 \mathrm{~min}\left[(S) \text {-enantiomer]. } 90 \% \text { ee. } \_\alpha\right]_{\mathrm{D}}^{20}$ $+56.2\left(c 0.94, \mathrm{CHCl}_{3}\right)$. The absolute configuration was assigned by analogy with Table 2 , entry 1.

${ }^{1} \mathrm{H} \mathrm{NMR}\left(\mathrm{CDCl}_{3}\right): \delta 7.24\left(\mathrm{dd},{ }^{3} J_{\mathrm{HH}}=8.7 \mathrm{~Hz}\right.$ and $\left.{ }^{3} \mathrm{~J}_{\mathrm{HF}}=5.3 \mathrm{~Hz}, 2 \mathrm{H}\right), 6.99\left(\mathrm{tt}^{3}\right)_{\mathrm{HH}}=8.7$ $\mathrm{Hz}$ and $\left.{ }^{3} J_{\mathrm{HF}}=2.2 \mathrm{~Hz}, 2 \mathrm{H}\right), 3.90\left(\mathrm{dd},{ }^{3} J_{\mathrm{HH}}=9.5 \mathrm{~Hz}\right.$ and $\left.6.1 \mathrm{~Hz}, 1 \mathrm{H}\right), 2.99\left(\mathrm{dd},{ }^{2} J_{\mathrm{HH}}=16.5\right.$ $\mathrm{Hz}$ and $\left.{ }^{3} J_{\mathrm{HH}}=9.5 \mathrm{~Hz}, 1 \mathrm{H}\right), 2.54\left(\mathrm{dd},{ }^{2} J_{\mathrm{HH}}=16.5 \mathrm{~Hz}\right.$ and $\left.{ }^{3} J_{\mathrm{HH}}=6.1 \mathrm{~Hz}, 1 \mathrm{H}\right), 1.40(\mathrm{~s}, 9 \mathrm{H})$, $1.38(\mathrm{~s}, 9 \mathrm{H}) .{ }^{13} \mathrm{C} \mathrm{NMR}\left(\mathrm{CDCl}_{3}\right): \delta 171.9,170.6,162.0\left(\mathrm{~d},{ }^{1} J_{\mathrm{CF}}=245 \mathrm{~Hz}\right), 134.2\left(\mathrm{~d},{ }^{4} J_{\mathrm{CF}}=3.6\right.$ $\mathrm{Hz}), 129.3\left(\mathrm{~d},{ }^{3} J_{\mathrm{CF}}=8.3 \mathrm{~Hz}\right), 115.5\left(\mathrm{~d},{ }^{2} J_{\mathrm{CF}}=21.1 \mathrm{~Hz}\right), 81.1,80.8,47.7,39.1,28.0,27.9$. Anal. Calcd for $\mathrm{C}_{18} \mathrm{H}_{25} \mathrm{FO}_{4}$ : C, 66.65; H, 7.77. Found: $\mathrm{C}, 66.53 ; \mathrm{H}, 7.85$.<smiles>CCCCOC(=O)C[C@H](C(=O)OCC)c1ccc2ccccc2c1</smiles>

Table 1, entry 9 (6d). White solid. 91\% yield. The ee was determined on a Daicel Chiralpak AD-H column with hexane : isopropanol $=95: 5$, flow $=1.0 \mathrm{~mL} / \mathrm{min}$. Retention times: $11.6 \mathrm{~min}\left[(R)\right.$-enantiomer], $26.6 \mathrm{~min}\left[(S) \text {-enantiomer]. } 87 \% \text { ee. } \_\right]^{20}{ }_{\mathrm{D}}$ $+61.5\left(c 1.62, \mathrm{CHCl}_{3}\right)$. The absolute configuration was assigned by analogy with Table 2 , entry 1.

${ }^{1} \mathrm{H}$ NMR $\left(\mathrm{CDCl}_{3}\right): \delta$ 7.84-7.70 $(\mathrm{m}, 4 \mathrm{H}), 7.50-7.39(\mathrm{~m}, 3 \mathrm{H}), 4.10\left(\mathrm{dd},{ }^{3} \mathrm{~J}_{\mathrm{HH}}=9.9 \mathrm{~Hz}\right.$ and $5.7 \mathrm{~Hz}, 1 \mathrm{H}), 3.12\left(\mathrm{dd},{ }^{2} J_{\mathrm{HH}}=16.6 \mathrm{~Hz}\right.$ and $\left.{ }^{3} J_{\mathrm{HH}}=9.9 \mathrm{~Hz}, 1 \mathrm{H}\right), 2.64\left(\mathrm{dd},{ }^{2} J_{\mathrm{HH}}=16.6 \mathrm{~Hz}\right.$ and $\left.{ }^{3} \mathrm{~J}_{\mathrm{HH}}=5.7 \mathrm{~Hz}, 1 \mathrm{H}\right), 1.40(\mathrm{~s}, 9 \mathrm{H}), 1.39(\mathrm{~s}, 9 \mathrm{H}) .{ }^{13} \mathrm{C} \mathrm{NMR}\left(\mathrm{CDCl}_{3}\right): \delta 172.1,170.8,136.0$, $133.5,132.6,128.3,127.8,127.6,126.6,126.1,125.9,125.8,81.1,80.8,48.1,39.1,28.0,27.9$. Anal. Calcd for $\mathrm{C}_{22} \mathrm{H}_{28} \mathrm{O}_{4}$ : C, 74.13; $\mathrm{H}, 7.92$. Found: $\mathrm{C}, 73.84 ; \mathrm{H}, 7.92$.<smiles>CCCCOC(=O)C[C@H](C(=O)OCCC)c1ccccc1C</smiles>

Table 1, entry 10 (6e). Pale yellow oil. 80\% yield. The ee was determined on a Daicel Chiralcel OD-H column with hexane : isopropanol $=500: 1$, flow $=1.0 \mathrm{~mL} / \mathrm{min}$. Retention times: $8.0 \mathrm{~min}\left[(R)\right.$-enantiomer], $12.2 \mathrm{~min}\left[(S) \text {-enantiomer]. } 91 \% \text { ee. } \_\alpha\right]^{20}{ }_{\mathrm{D}}$ $+78.8\left(c 1.28, \mathrm{CHCl}_{3}\right)$. The absolute configuration was assigned by analogy with Table 2 , entry 1.

${ }^{1} \mathrm{H}$ NMR $\left(\mathrm{CDCl}_{3}\right): \delta 7.22-7.08(\mathrm{~m}, 4 \mathrm{H}), 4.21\left(\mathrm{dd},{ }^{3} \mathrm{~J}_{\mathrm{HH}}=9.9 \mathrm{~Hz}\right.$ and $\left.5.6 \mathrm{~Hz}, 1 \mathrm{H}\right), 3.00$ 
$\left(\mathrm{dd},{ }^{2} J_{\mathrm{HH}}=16.5 \mathrm{Hs}\right.$ and $\left.{ }^{3} J_{\mathrm{HH}}=9.9 \mathrm{~Hz}, 1 \mathrm{H}\right), 2.50\left(\mathrm{dd},{ }^{2} J_{\mathrm{HH}}=16.5 \mathrm{~Hz}\right.$ and ${ }^{3} J_{\mathrm{HH}}=5.6 \mathrm{~Hz}$, $1 \mathrm{H}), 2.41(\mathrm{~s}, 3 \mathrm{H}), 1.39(\mathrm{~s}, 9 \mathrm{H}), 1.37(\mathrm{~s}, 9 \mathrm{H}) .{ }^{13} \mathrm{C} \mathrm{NMR}\left(\mathrm{CDCl}_{3}\right): \delta 172.4,171.1,137.0,136.1$, $130.5,127.0,126.5,126.2,80.8,80.6,44.0,38.4,28.0,27.9,19.8$. Anal. Calcd for $\mathrm{C}_{19} \mathrm{H}_{28} \mathrm{O}_{4}$ : C, 71.22; H, 8.81. Found: C, 71.44; H, 9.03.

\section{General Procedure for Table 2.}

A solution of $\left[\mathrm{RhCl}\left(\mathrm{C}_{2} \mathrm{H}_{4}\right)_{2}\right]_{2}(1.9 \mathrm{mg}, 9.8 \mu \mathrm{mol} \mathrm{Rh})$ and chiral ligand $(11 \mu \mathrm{mol})$ in 1,4-dioxane $(1.0 \mathrm{~mL})$ was stirred for $10 \mathrm{~min}$ at room temperature. $\mathrm{KOH}(0.20 \mathrm{~mL}, 20$ $\mu \mathrm{mol} ; 0.1 \mathrm{M}$ aqueous) was then added to it, and the resulting solution was stirred for additional $10 \mathrm{~min}$ at room temperature. After addition of $\operatorname{ArB}(\mathrm{OH})_{2}(0.60 \mathrm{mmol})$ and stirring for $5 \mathrm{~min}$, this mixture was transferred to a solution of maleimide $(0.20 \mathrm{mmol})$ in 1,4-dioxane $(1.0 \mathrm{~mL})$. The resulting mixture was stirred for $3 \mathrm{~h}$ at $50{ }^{\circ} \mathrm{C}$, and was then passed through a pad of silica gel with $\mathrm{Et}_{2} \mathrm{O}$, and the solvent was removed under vacuum. The residue was purified by silica gel PTLC with EtOAc/hexane to afford the desired 1,4-adduct 8.

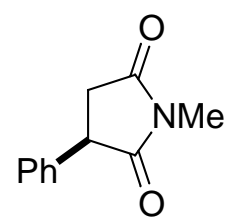

Table 2, entry 6 (8a). (CAS 86-34-0) 88\% conversion. The ee was determined on a Daicel Chiralpak AD-H column with hexane : isopropanol $=90: 10$, flow $=1.0$ $\mathrm{mL} / \mathrm{min}$. Retention times: $15.2 \mathrm{~min}$ [(R)-enantiomer], $18.1 \mathrm{~min}[(S)$-enantiomer]. 85\% ee. The absolute configuration was assigned by analogy with Table 2, entry 8 .

${ }^{1} \mathrm{H} \mathrm{NMR}\left(\mathrm{CDCl}_{3}\right): \delta 7.36\left(\mathrm{t},{ }^{3} \mathrm{~J}_{\mathrm{HH}}=7.2 \mathrm{~Hz}, 2 \mathrm{H}\right), 7.30\left(\mathrm{tt},{ }^{3} \mathrm{~J}_{\mathrm{HH}}=7.2 \mathrm{~Hz}\right.$ and ${ }^{4} J_{\mathrm{HH}}=$ $1.3 \mathrm{~Hz}, 1 \mathrm{H}), 7.21\left(\mathrm{~d},{ }^{3} J_{\mathrm{HH}}=7.2 \mathrm{~Hz}, 2 \mathrm{H}\right), 4.02\left(\mathrm{dd},{ }^{3} \mathrm{~J}_{\mathrm{HH}}=9.5 \mathrm{~Hz}\right.$ and $\left.4.8 \mathrm{~Hz}, 1 \mathrm{H}\right), 3.20(\mathrm{dd}$, ${ }^{2} J_{\mathrm{HH}}=18.4 \mathrm{~Hz}$ and $\left.{ }^{3} J_{\mathrm{HH}}=9.5 \mathrm{~Hz}, 1 \mathrm{H}\right), 3.06(\mathrm{~s}, 3 \mathrm{H}), 2.83\left(\mathrm{dd},{ }^{2} J_{\mathrm{HH}}=18.4 \mathrm{~Hz}\right.$ and ${ }^{3} \mathrm{~J}_{\mathrm{HH}}=$ $4.8 \mathrm{~Hz}, 1 \mathrm{H}) .{ }^{13} \mathrm{C}$ NMR $\left(\mathrm{CDCl}_{3}\right): \delta 177.8,176.2,137.1,129.2,127.9,127.4,45.9,37.1,25.2$.

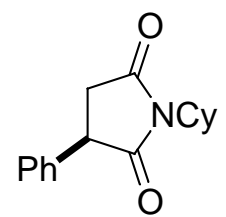

Table 2, entry 7 (8b). (CAS 139477-41-1) White solid. 85\% yield. The ee was determined on a Daicel Chiralcel OD-H column with hexane : isopropanol $=90: 10$, flow $=1.0 \mathrm{~mL} / \mathrm{min}$. Retention times: $9.3 \mathrm{~min}[(S)$-enantiomer], $11.1 \mathrm{~min}[(R)-$ enantiomer]. $87 \%$ ee. $\left.\_\alpha\right]_{D}^{20}-34.0\left(c^{2} 0.82, \mathrm{CHCl}_{3}\right)$. The absolute configuration was assigned by analogy with Table 2 , entry 8 .

${ }^{1} \mathrm{H}$ NMR $\left(\mathrm{CDCl}_{3}\right): \delta 7.35\left(\mathrm{t},{ }^{3} J_{\mathrm{HH}}=7.3 \mathrm{~Hz}, 2 \mathrm{H}\right), 7.29\left(\mathrm{t},{ }^{3} \mathrm{~J}_{\mathrm{HH}}=7.3 \mathrm{~Hz}, 1 \mathrm{H}\right), 7.19(\mathrm{~d}$, $\left.{ }^{3} \mathrm{~J}_{\mathrm{HH}}=7.3 \mathrm{~Hz}, 2 \mathrm{H}\right), 4.03\left(\mathrm{tt},{ }^{3} \mathrm{~J}_{\mathrm{HH}}=12.7 \mathrm{~Hz}\right.$ and $\left.3.7 \mathrm{~Hz}, 1 \mathrm{H}\right), 3.93\left(\mathrm{dd},{ }^{3} \mathrm{~J}_{\mathrm{HH}}=9.7 \mathrm{~Hz}\right.$ and $4.5 \mathrm{~Hz}, 1 \mathrm{H}), 3.12\left(\mathrm{dd},{ }^{2} J_{\mathrm{HH}}=18.4 \mathrm{~Hz}\right.$ and $\left.{ }^{3} J_{\mathrm{HH}}=9.7 \mathrm{~Hz}, 1 \mathrm{H}\right), 2.74\left(\mathrm{dd},{ }^{2} J_{\mathrm{HH}}=18.4 \mathrm{~Hz}\right.$ and $\left.{ }^{3} \mathrm{JHH}_{\mathrm{HH}}=4.5 \mathrm{~Hz}, 1 \mathrm{H}\right), 2.24-2.13(\mathrm{~m}, 2 \mathrm{H}), 1.88-1.79(\mathrm{~m}, 2 \mathrm{H}), 1.70-1.58(\mathrm{~m}, 3 \mathrm{H}), 1.41-1.15(\mathrm{~m}$, $3 \mathrm{H}) .{ }^{13} \mathrm{C} \mathrm{NMR}\left(\mathrm{CDCl}_{3}\right): \delta 177.8,176.3,138.0,129.1,127.8,127.2,52.0,45.6,37.1,28.9,28.7$, $25.84,25.82,25.0$. 


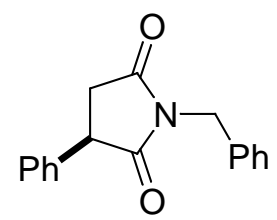

Table 2, entry 8 (8c). (CAS 42856-57-5) White solid. 88\% yield. The ee was determined on a Daicel Chiralcel OD-H column with hexane : isopropanol $=90: 10$, flow $=1.0 \mathrm{~mL} / \mathrm{min}$. Retention times: $21.0 \mathrm{~min}[(S)$-enantiomer], $25.7 \mathrm{~min}[(R)-$ enantiomer]. $69 \%$ ee. $\left.\_\alpha\right]_{D}^{20}-33.7$ (c 1.20, $\mathrm{CHCl}_{3}$ ). The absolute configuration was determined by comparison of the optical rotation with that reported in the literature. ${ }^{6}$

${ }^{1} \mathrm{H} \mathrm{NMR}\left(\mathrm{CDCl}_{3}\right): \delta 7.40\left(\mathrm{~d},{ }^{3} \mathrm{~J}_{\mathrm{HH}}=6.6 \mathrm{~Hz}, 2 \mathrm{H}\right), 7.36-7.26(\mathrm{~m}, 6 \mathrm{H}), 7.15\left(\mathrm{~d},{ }^{3} J_{\mathrm{HH}}=6.8\right.$ $\mathrm{Hz}, 2 \mathrm{H}), 4.72\left(\mathrm{~d},{ }^{2} J_{\mathrm{HH}}=14.1 \mathrm{~Hz}, 1 \mathrm{H}\right), 4.70\left(\mathrm{~d},{ }^{2} J_{\mathrm{HH}}=14.1 \mathrm{~Hz}, 1 \mathrm{H}\right), 4.01\left(\mathrm{dd},{ }^{3} J_{\mathrm{HH}}=9.6 \mathrm{~Hz}\right.$ and $4.8 \mathrm{~Hz}, 1 \mathrm{H}), 3.19\left(\mathrm{dd},{ }^{2} J_{\mathrm{HH}}=18.5 \mathrm{~Hz}\right.$ and $\left.{ }^{3} J_{\mathrm{HH}}=9.6 \mathrm{~Hz}, 1 \mathrm{H}\right), 2.81\left(\mathrm{dd},{ }^{2} J_{\mathrm{HH}}=18.5 \mathrm{~Hz}\right.$ and $\left.{ }^{3} J_{\mathrm{HH}}=4.8 \mathrm{~Hz}, 1 \mathrm{H}\right) .{ }^{13} \mathrm{C} \mathrm{NMR}\left(\mathrm{CDCl}_{3}\right): \delta 177.4,175.7,137.2,135.8,129.2,128.8,128.7$, $128.0,127.9,127.4,45.9,42.8,37.2$.<smiles>COc1ccc(C2CC(=O)N(C)C2=O)cc1</smiles>

Table 2, entry 9 (8d). White solid. $77 \%$ yield. The ee was determined on a Daicel Chiralcel OD-H column with hexane : isopropanol $=90: 10$, flow $=1.0 \mathrm{~mL} / \mathrm{min}$. Retention times: 13.2 min [(R)-enantiomer], 16.0 min $\left[(S) \text {-enantiomer]. } 84 \% \text { ee. } \_\right]^{20}{ }_{\mathrm{D}}$ $-34.6\left(c 1.13, \mathrm{CHCl}_{3}\right)$. The absolute configuration was assigned by analogy with Table 2 , entry 8.

${ }^{1} \mathrm{H} \mathrm{NMR}\left(\mathrm{CDCl}_{3}\right): \delta 7.10\left(\mathrm{~d},{ }^{3} J_{\mathrm{HH}}=8.7 \mathrm{~Hz}, 2 \mathrm{H}\right), 6.88\left(\mathrm{~d},{ }^{3} J_{\mathrm{HH}}=8.7 \mathrm{~Hz}, 2 \mathrm{H}\right), 4.02(\mathrm{tt}$, ${ }^{3} J_{\mathrm{HH}}=12.4 \mathrm{~Hz}$ and $\left.3.6 \mathrm{~Hz}, 1 \mathrm{H}\right), 3.87\left(\mathrm{dd},{ }^{3} J_{\mathrm{HH}}=9.6 \mathrm{~Hz}\right.$ and $\left.4.6 \mathrm{~Hz}, 1 \mathrm{H}\right), 3.79(\mathrm{~s}, 3 \mathrm{H}), 3.10$ $\left(\mathrm{dd},{ }^{2} J_{\mathrm{HH}}=18.4 \mathrm{~Hz}\right.$ and $\left.{ }^{3} J_{\mathrm{HH}}=9.6 \mathrm{~Hz}, 1 \mathrm{H}\right), 2.70\left(\mathrm{dd},{ }^{2} J_{\mathrm{HH}}=18.4 \mathrm{~Hz}\right.$ and ${ }^{3} J_{\mathrm{HH}}=4.6 \mathrm{~Hz}$, $1 \mathrm{H}), 2.24-2.12(\mathrm{~m}, 2 \mathrm{H}), 1.87-1.78(\mathrm{~m}, 2 \mathrm{H}), 1.69-1.57(\mathrm{~m}, 3 \mathrm{H}), 1.37-1.16(\mathrm{~m}, 3 \mathrm{H}) .{ }^{13} \mathrm{C}$ NMR $\left(\mathrm{CDCl}_{3}\right): \delta 178.1,176.4,159.2,129.7,128.3,114.6,55.3,52.0,44.8,37.2,28.9,28.8,25.84$, 25.82, 25.0. Anal. Calcd for $\mathrm{C}_{17} \mathrm{H}_{21} \mathrm{NO}_{3}$ : C, 71.06; H, 7.37. Found: $\mathrm{C}, 70.88 ; \mathrm{H}, 7.41$.

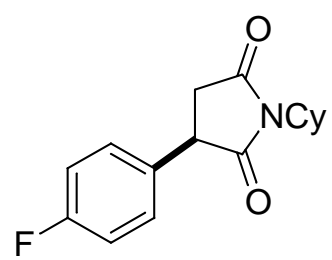

Table 2, entry 10 (8e). White solid. 88\% yield. The ee was determined on a Daicel Chiralpak AD-H column with hexane : isopropanol $=90: 10$, flow $=1.0 \mathrm{~mL} / \mathrm{min}$. Retention times: $13.4 \mathrm{~min}\left[(R)\right.$-enantiomer], $53.5 \mathrm{~min}\left[(S) \text {-enantiomer]. } 86 \% \text { ee. } \_\right]^{20}{ }_{\mathrm{D}}$ $-36.5\left(c 0.96, \mathrm{CHCl}_{3}\right)$. The absolute configuration was assigned by analogy with Table 2 , entry 8.

${ }^{1} \mathrm{H}$ NMR $\left(\mathrm{CDCl}_{3}\right): \delta 7.17\left(\mathrm{dd},{ }^{3} \mathrm{~J}_{\mathrm{HH}}=8.6 \mathrm{~Hz}\right.$ and $\left.{ }^{3} \mathrm{~J}_{\mathrm{HF}}=5.2 \mathrm{~Hz}, 2 \mathrm{H}\right), 7.05\left(\mathrm{t}^{3}{ }^{3} \mathrm{~J}=8.6 \mathrm{~Hz}\right.$, $2 \mathrm{H}), 4.03\left(\mathrm{tt},{ }^{3} \mathrm{~J}_{\mathrm{HH}}=12.5 \mathrm{~Hz}\right.$ and $\left.3.7 \mathrm{~Hz}, 1 \mathrm{H}\right), 3.92\left(\mathrm{dd},{ }^{3} \mathrm{~J}_{\mathrm{HH}}=9.7 \mathrm{~Hz}\right.$ and $\left.4.7 \mathrm{~Hz}, 1 \mathrm{H}\right), 3.12$

\footnotetext{
${ }^{6}$ Bettoni, G.; Franchini, C.; Morlacchi, F.; Tangari, N.; Tortorella, V. J. Org. Chem. 1976, 41,2780 .
} 
$\left(\mathrm{dd},{ }^{2} J_{\mathrm{HH}}=18.4 \mathrm{~Hz}\right.$ and $\left.{ }^{3} J_{\mathrm{HH}}=9.7 \mathrm{~Hz}, 1 \mathrm{H}\right), 2.70\left(\mathrm{dd},{ }^{2} J_{\mathrm{HH}}=18.4 \mathrm{~Hz}\right.$ and ${ }^{3} \mathrm{~J}_{\mathrm{HH}}=4.7 \mathrm{~Hz}$, $1 \mathrm{H}), 2.23-2.13(\mathrm{~m}, 1 \mathrm{H}), 1.87-1.79(\mathrm{~m}, 2 \mathrm{H}), 1.69-1.58(\mathrm{~m}, 3 \mathrm{H}), 1.38-1.16(\mathrm{~m}, 3 \mathrm{H}) .{ }^{13} \mathrm{C} \mathrm{NMR}$ $\left(\mathrm{CDCl}_{3}\right): \delta 177.6,176.0,162.2\left(\mathrm{~d},{ }^{1} J_{\mathrm{CF}}=246 \mathrm{~Hz}\right), 133.4\left(\mathrm{~d},{ }^{4} J_{\mathrm{CF}}=3.6 \mathrm{~Hz}\right), 128.9\left(\mathrm{~d},{ }^{3} J_{\mathrm{CF}}=8.1\right.$ $\mathrm{Hz}), 116.1\left(\mathrm{~d},{ }^{2} J_{\mathrm{CF}}=21.6 \mathrm{~Hz}\right), 52.1,44.8,37.0,28.9,28.8,25.82,25.80,25.0$. Anal. Calcd for $\mathrm{C}_{16} \mathrm{H}_{18} \mathrm{FNO}_{2}$ : C, 69.80; $\mathrm{H}, 6.59$. Found: $\mathrm{C}, 69.71 ; \mathrm{H}, 6.59$.<smiles>O=C1CC(c2cccc(Cl)c2)C(=O)N1O</smiles>

Table 2, entry 11 (8f). White solid. 92\% yield. The ee was determined on a Daicel Chiralpak AD-H column with hexane : isopropanol $=90: 10$, flow $=1.0 \mathrm{~mL} / \mathrm{min}$. Retention times: $19.1 \mathrm{~min}\left[(R)\right.$-enantiomer], $56.6 \mathrm{~min}\left[(S) \text {-enantiomer]. } 82 \% \text { ee. } \_\right]^{20}{ }_{\mathrm{D}}$ $-26.5\left(c 1.00, \mathrm{CHCl}_{3}\right)$. The absolute configuration was assigned by analogy with Table 2 , entry 8.

${ }^{1} \mathrm{H}$ NMR $\left(\mathrm{CDCl}_{3}\right): \delta$ 7.32-7.27 (m, 2H), 7.21-7.18 (m, 1H), 7.10-7.06 (m, 1H), $4.03(\mathrm{tt}$, ${ }^{3} J_{\mathrm{HH}}=12.4 \mathrm{~Hz}$ and $\left.3.6 \mathrm{~Hz}, 1 \mathrm{H}\right), 3.91\left(\mathrm{dd},{ }^{3} J_{\mathrm{HH}}=9.7 \mathrm{~Hz}\right.$ and $\left.4.7 \mathrm{~Hz}, 1 \mathrm{H}\right), 3.14\left(\mathrm{dd},{ }^{2} J_{\mathrm{HH}}=\right.$ $18.4 \mathrm{~Hz}$ and $\left.{ }^{3} \mathrm{~J}_{\mathrm{HH}}=9.7 \mathrm{~Hz}, 1 \mathrm{H}\right), 2.72\left(\mathrm{dd},{ }^{2} J_{\mathrm{HH}}=18.4 \mathrm{~Hz}\right.$ and $\left.{ }^{3} J_{\mathrm{HH}}=4.7 \mathrm{~Hz}, 1 \mathrm{H}\right), 2.24-2.13$ $(\mathrm{m}, 2 \mathrm{H}), 1.88-1.80(\mathrm{~m}, 2 \mathrm{H}), 1.66-1.56(\mathrm{~m}, 3 \mathrm{H}), 1.38-1.17(\mathrm{~m}, 3 \mathrm{H}) .{ }^{13} \mathrm{C} \mathrm{NMR}\left(\mathrm{CDCl}_{3}\right): \delta$ 177.1, 175.8, 139.5, 135.0, 130.4, 128.1, 127.7, 125.4, 52.2, 45.2, 36.9, 28.9, 28.8, 25.87, 25.85, 25.0. Anal. Calcd for $\mathrm{C}_{16} \mathrm{H}_{18} \mathrm{ClNO}_{2}$ : C, 65.86; $\mathrm{H}, 6.22$. Found: $\mathrm{C}, 65.61 ; \mathrm{H}, 6.17$.<smiles>CN1C(=O)CC(c2ccc3ccccc3c2)C1=O</smiles>

Table 2, entry 12 (8g). White solid. 93\% yield. The ee was determined on a Daicel Chiralpak AD-H column with hexane : isopropanol $=90: 10$, flow $=1.0 \mathrm{~mL} / \mathrm{min}$. Retention times: $19.6 \mathrm{~min}\left[(R)\right.$-enantiomer], $96.7 \mathrm{~min}\left[(S) \text {-enantiomer]. } 84 \% \text { ee. } \_\right]^{20}{ }_{\mathrm{D}}$ $-46.4\left(c 1.11, \mathrm{CHCl}_{3}\right)$. The absolute configuration was assigned by analogy with Table 2 , entry 8.

${ }^{1} \mathrm{H} \mathrm{NMR}\left(\mathrm{CDCl}_{3}\right): \delta$ 7.86-7.76 $(\mathrm{m}, 3 \mathrm{H}), 7.67(\mathrm{~s}, 1 \mathrm{H}), 7.52-7.45(\mathrm{~m}, 2 \mathrm{H}), 7.28-7.23(\mathrm{~m}$, $1 \mathrm{H}), 4.14-4.02(\mathrm{~m}, 2 \mathrm{H}), 3.19\left(\mathrm{dd},{ }^{2} J_{\mathrm{HH}}=18.4 \mathrm{~Hz}\right.$ and $\left.{ }^{3} \mathrm{~J}_{\mathrm{HH}}=9.6 \mathrm{~Hz}, 1 \mathrm{H}\right), 2.84\left(\mathrm{dd},{ }^{2} J_{\mathrm{HH}}=\right.$ $18.4 \mathrm{~Hz}$ and $\left.{ }^{3} \mathrm{~J}_{\mathrm{HH}}=4.4 \mathrm{~Hz}, 1 \mathrm{H}\right), 2.28-2.15(\mathrm{~m}, 2 \mathrm{H}), 1.89-1.80(\mathrm{~m}, 2 \mathrm{H}), 1.72-1.62(\mathrm{~m}, 3 \mathrm{H})$, 1.40-1.17 $(\mathrm{m}, 3 \mathrm{H}) .{ }^{13} \mathrm{C}$ NMR $\left(\mathrm{CDCl}_{3}\right): \delta 177.8,176.3,135.0,133.4,132.8,129.3,127.8$, 127.7, 126.6, 126.5, 126.3, 124.7, 52.2, 45.8, 37.1, 29.0, 28.8, 25.89, 25.87, 25.0. Anal. Calcd for $\mathrm{C}_{20} \mathrm{H}_{21} \mathrm{NO}_{2}$ : C, 78.15; $\mathrm{H}, 6.89$. Found: $\mathrm{C}, 77.96 ; \mathrm{H}, 6.69$.<smiles>Cc1ccccc1C1CC(=O)N(Cl)C1=O</smiles>

Table 2, entry 13 (8h). Colorless viscous oil. 95\% yield. The ee was determined on a Daicel Chiralpak AD-H column with hexane : isopropanol $=95: 5$, flow $=0.5$ 
$\mathrm{mL} / \mathrm{min}$. Retention time: $37.8 \mathrm{~min}$ [(R)-enantiomer], $90.3 \mathrm{~min}[(S)$-enantiomer]. $92 \%$ ee. $\left.\_\right]^{20}{ }_{D}+17.4\left(c 0.91, \mathrm{CHCl}_{3}\right)$. The absolute configuration was assigned by analogy with Table 2, entry 8 .

${ }^{1} \mathrm{H}$ NMR $\left(\mathrm{CDCl}_{3}\right): \delta$ 7.22-7.15 (m, 3H), 7.00-6.95 (m, $\left.1 \mathrm{H}\right), 4.15\left(\mathrm{dd},{ }^{3} \mathrm{~J}_{\mathrm{HH}}=9.7 \mathrm{~Hz}\right.$ and $4.8 \mathrm{~Hz}, 1 \mathrm{H}), 4.07\left(\mathrm{tt},{ }^{3} \mathrm{~J}_{\mathrm{HH}}=12.4 \mathrm{~Hz}\right.$ and $\left.3.8 \mathrm{~Hz}, 1 \mathrm{H}\right), 3.14\left(\mathrm{dd},{ }^{2} J_{\mathrm{HH}}=18.4 \mathrm{~Hz}\right.$ and ${ }^{3} \mathrm{~J}_{\mathrm{HH}}=$ $9.7 \mathrm{~Hz}, 1 \mathrm{H}), 2.61\left(\mathrm{dd},{ }^{2} J_{\mathrm{HH}}=18.4 \mathrm{~Hz}\right.$ and $\left.{ }^{3} J_{\mathrm{HH}}=4.8 \mathrm{~Hz}, 1 \mathrm{H}\right), 2.38(\mathrm{~s}, 3 \mathrm{H}), 2.27-2.16(\mathrm{~m}$, $2 \mathrm{H}), 1.89-1.80(\mathrm{~m}, 2 \mathrm{H}), 1.71-1.61(\mathrm{~m}, 3 \mathrm{H}), 1.40-1.18(\mathrm{~m}, 3 \mathrm{H}) .{ }^{13} \mathrm{C}$ NMR $\left(\mathrm{CDCl}_{3}\right): \delta 178.3$, 176.3, 136.7, 136.3, 131.1, 127.8, 126.8, 126.4, 52.1, 42.8, 37.0, 28.9, 28.8, 25.91, 25.89, 25.1, 19.8. Anal. Calcd for $\mathrm{C}_{17} \mathrm{H}_{21} \mathrm{NO}_{2}$ : $\mathrm{C}, 75.25 ; \mathrm{H}, 7.80$. Found: $\mathrm{C}, 75.00 ; \mathrm{H}, 7.76$.

\section{X-ray Crystal Structure of $[\mathrm{RhCl}((R, R)-5 a)]_{2}$}

\section{Data Collection}

A yellow prism crystal of $\mathrm{C}_{42} \mathrm{H}_{40} \mathrm{Cl}_{2} \mathrm{Rh}_{2}$ having approximate dimensions of $0.15 \mathrm{x}$ $0.10 \times 0.05 \mathrm{~mm}$ was mounted on a glass fiber. All measurements were made on a Rigaku RAXIS RAPID imaging plate area detector with graphite monochromated Mo$\mathrm{K} \alpha$ radiation.

Indexing was performed from $3^{\circ}$ oscillations that were exposed for 90 seconds. The crystal-to-detector distance was $127.40 \mathrm{~mm}$.

Cell constants and an orientation matrix for data collection corresponded to a Ccentered monoclinic cell with dimensions:

$$
\begin{aligned}
& \mathrm{a}=28.551(9) \AA \\
& \mathrm{b}=5.731(2) \AA \quad \beta=107.15(2)^{\circ} \\
& \mathrm{c}=12.165(3) \AA \\
& \mathrm{V}=1901.9(1) \AA^{3}
\end{aligned}
$$

For $\mathrm{Z}=2$ and F.W. $=821.50$, the calculated density is $1.43 \mathrm{~g} / \mathrm{cm}^{3}$. Based on the systematic absences of:

$$
\text { hkl: } \mathrm{h}+\mathrm{k} \pm 2 \mathrm{n}
$$

packing considerations, a statistical analysis of intensity distribution, and the successful solution and refinement of the structure, the space group was determined to be:

\section{C2 (\#5)}

The data were collected at a temperature of $-150 \pm 1{ }^{\circ} \mathrm{C}$ to a maximum $2 \theta$ value of $54.9^{\circ}$. A total of 44 oscillation images were collected. A sweep of data was done using $\omega$ scans from 130.0 to $190.0^{\circ}$ in $5.0^{\circ}$ step, at $\chi=45.0^{\circ}$ and $\phi=0.0^{\circ}$. The exposure rate was $150.0\left[\mathrm{sec} . /^{\circ}\right.$ ]. A second sweep was performed using $\omega$ scans from 0.0 to $160.0^{\circ}$ in $5.0^{\circ}$ step, at $\chi=45.0^{\circ}$ and $\phi=180.0^{\circ}$. The exposure rate was 150.0 [sec. $/^{\circ}$ ]. The crystal-todetector distance was $127.40 \mathrm{~mm}$. Readout was performed in the $0.100 \mathrm{~mm}$ pixel mode. 


\section{Data Reduction}

Of the 9385 reflections that were collected, 2401 were unique ( $\operatorname{Rint}=0.033$ ); equivalent reflections were merged.

The linear absorption coefficient, $\mu$, for Mo-K $\alpha$ radiation is $10.3 \mathrm{~cm}^{-1}$. The data were corrected for Lorentz and polarization effects.

\section{Structure Solution and Refinement}

The structure was solved by direct methods ${ }^{7}$ and expanded using Fourier techniques. ${ }^{8}$ Some non-hydrogen atoms were refined anisotropically, while the rest were refined isotropically. Hydrogen atoms were refined using the riding model. The final cycle of full-matrix least-squares refinement ${ }^{9}$ on $\mathrm{F}$ was based on 2009 observed reflections $(\mathrm{I}>3.00 \sigma(\mathrm{I}))$ and 224 variable parameters and converged (largest parameter shift was 0.00 times its esd) with unweighted and weighted agreement factors of:

$$
\begin{gathered}
\mathrm{R}=\Sigma|| \mathrm{FO}_{0}|-| \mathrm{FC}_{\mathrm{C}}|| / \Sigma|\mathrm{FO}|=0.038 \\
\mathrm{R}_{\mathrm{W}}=\left[\Sigma \mathrm{w}\left(\left|\mathrm{FO}_{\mathrm{O}}\right|-\left|\mathrm{FC}_{\mathrm{C}}\right|\right)^{2} / \Sigma \mathrm{w} \mathrm{FO}^{2}\right]^{1 / 2}=0.063
\end{gathered}
$$

The standard deviation of an observation of unit weight ${ }^{10}$ was 0.88 . Unit weights were used. Plots of $\Sigma \mathrm{w}\left(\left|\mathrm{Fo}_{\mid}\right|-\left|\mathrm{FC}_{\mathrm{C}}\right|\right)^{2}$ versus $\left|\mathrm{Fo}_{\mathrm{O}}\right|$, reflection order in data collection, $\sin \theta / \lambda$ and various classes of indices showed no unusual trends. The maximum and minimum peaks on the final difference Fourier map corresponded to 3.01 and -0.43 $\mathrm{e}^{-} / \AA^{3}$, respectively.

Neutral atom scattering factors were taken from Cromer and Waber. ${ }^{11}$ Anomalous dispersion effects were included in Fcalc; ${ }^{12}$ the values for $\Delta \mathrm{f}^{\prime}$ and $\Delta \mathrm{f}^{\prime \prime}$ were those of Creagh and McAuley. ${ }^{13}$ The values for the mass attenuation coefficients are those of Creagh and Hubbell. ${ }^{14}$ All calculations were performed using the CrystalStructure ${ }^{15,16}$ crystallographic software package.

${ }^{7}$ SIR92: Altomare, A.; Cascarano, G.; Giacovazzo, C.; Guagliardi, A.; Burla, M.; Polidori, G.; Camalli, M. J. Appl. Cryst. 1994, 27, 435.

${ }^{8}$ DIRDIF99: Beurskens, P. T.; Admiraal, G.; Beurskens, G.; Bosman, W. P.; de Gelder, R.; Israel, R.; Smits, J. M. M. The DIRDIF-99 program system, Technical Report of the Crystallography Laboratory, University of Nijmegen, The Netherlands (1999).

${ }^{9}$ Least Squares function minimized:

$$
\Sigma w\left(\left|\mathrm{~F}_{\mathrm{O}}\right|-\left|\mathrm{F}_{\mathrm{C}}\right|\right)^{2} \text { where } \mathrm{w}=\text { Least Squares weights. }
$$

${ }^{10}$ Standard deviation of an observation of unit weight:

$$
\left[\Sigma w\left(\left|\mathrm{~F}_{\mathrm{O}}\right|-\left|\mathrm{F}_{\mathrm{C}}\right|\right)^{2} /\left(\mathrm{N}_{\mathrm{O}}-\mathrm{N}_{\mathrm{V}}\right)\right]^{1 / 2}
$$

where: $\mathrm{N}_{\mathrm{O}}=$ number of observations, $\mathrm{N}_{\mathrm{V}}=$ number of variables

${ }^{11}$ Cromer, D. T.; Waber, J. T. "International Tables for X-ray Crystallography", Vol. IV, The Kynoch Press, Birmingham, England, Table 2.2 A (1974).

${ }^{12}$ Ibers, J. A.; Hamilton, W. C. Acta Crystallogr. 1964, 17, 781.

${ }^{13}$ Creagh, D. C.; McAuley, W. J. "International Tables for Crystallography", Vol C, (A.J.C. Wilson, ed.), Kluwer Academic Publishers, Boston, Table 4.2.6.8, pages 219-222 (1992).

${ }^{14}$ Creagh, D. C.; Hubbell, J. H. "International Tables for Crystallography", Vol C, (A.J.C. Wilson, ed.), Kluwer Academic Publishers, Boston, Table 4.2.4.3, pages 200-206 (1992).

${ }^{15}$ CrystalStructure 3.00: Crystal Structure Analysis Package, Rigaku and Rigaku/MSC (2000-2002). 

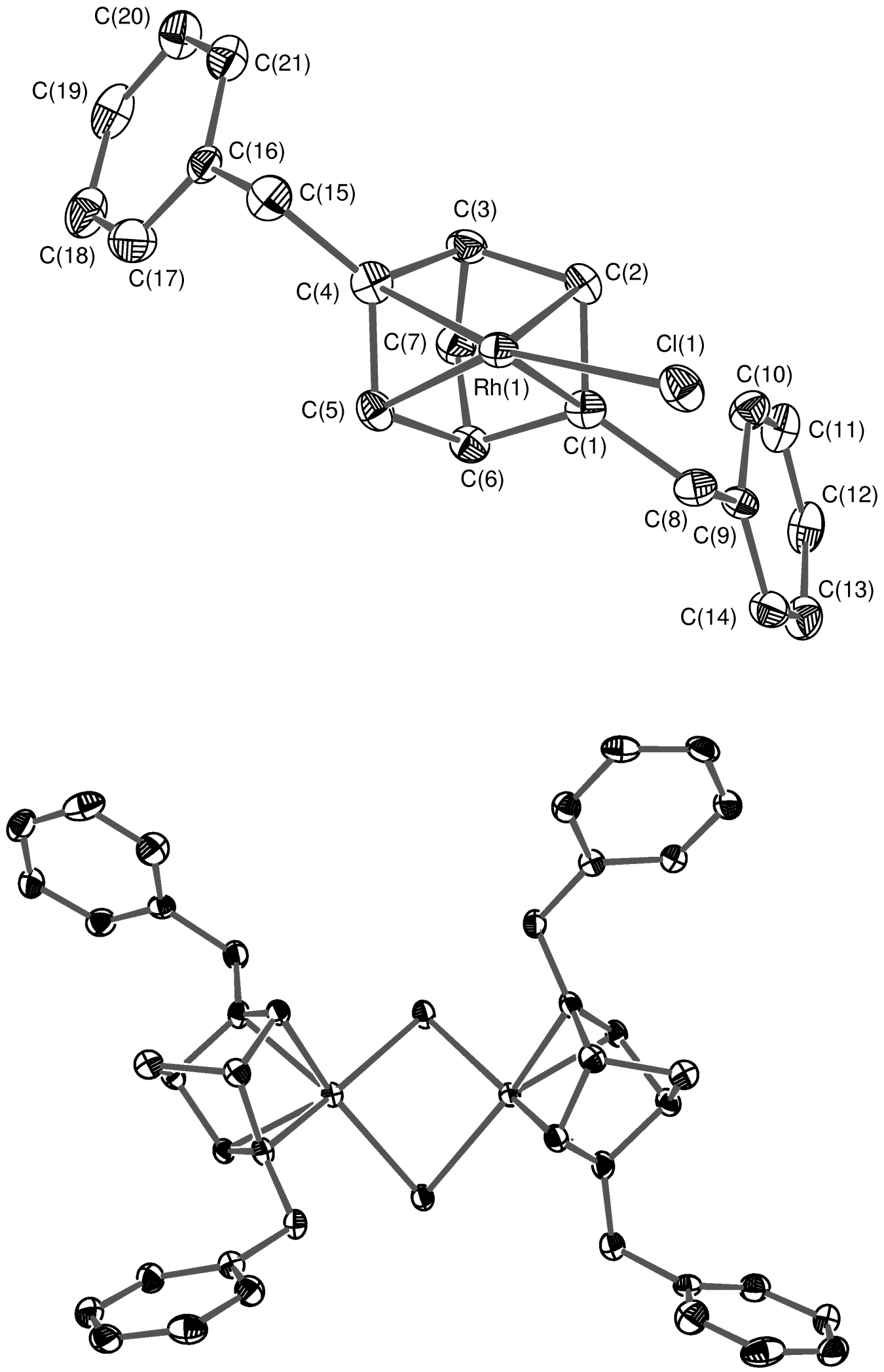

${ }^{16}$ CRYSTALS Issue 10: Watkin, D. J.; Prout, C. K.; Carruthers, J. R.; Betteridge, P. W. Chemical Crystallography Laboratory, Oxford, UK. 


\section{Experimental Details}

\section{A. Crystal Data}

Empirical Formula

Formula Weight

Crystal Color, Habit

Crystal Dimensions

Crystal System

Lattice Type

Indexing Images

Detector Position

Pixel Size

Lattice Parameters

Space Group

$Z$ value

Dcalc

F000

$\mu(\operatorname{MoK} \alpha)$
$\mathrm{C}_{42} \mathrm{H}_{40} \mathrm{Cl}_{2} \mathrm{Rh}_{2}$

821.50

yellow, prism

$0.15 \times 0.10 \times 0.05 \mathrm{~mm}$

monoclinic

C-centered

3 oscillations @ 90.0 seconds

$127.40 \mathrm{~mm}$

$0.100 \mathrm{~mm}$

$$
\begin{aligned}
& \mathrm{a}=28.551(9) \AA \\
& \mathrm{b}=5.731(2) \AA \\
& \mathrm{c}=12.165(3) \AA \\
& \beta=107.15(2)^{\circ} \\
& \mathrm{V}=1901.9(1) \AA^{3}
\end{aligned}
$$

C2 (\#5)

2

$1.434 \mathrm{~g} / \mathrm{cm}^{3}$

832.00

$10.33 \mathrm{~cm}^{-1}$ 


\section{B. Intensity Measurements}

Diffractometer

Radiation

Detector Aperture

Data Images

$\omega$ oscillation Range $(\chi=45.0, \phi=0.0)$

Exposure Rate

$\omega$ oscillation Range $(\chi=45.0, \phi=180.0)$

Exposure Rate

Detector Position

Pixel Size

$2 \theta_{\max }$

No. of Reflections Measured

Corrections
Rigaku RAXIS-RAPID

$\operatorname{MoK} \alpha(\lambda=0.71075 \approx)$

graphite monochromated

$270 \mathrm{~mm} \times 256 \mathrm{~mm}$

44 exposures

$130.0-190.0^{\circ}$

$150.0 \mathrm{sec} . /^{\circ}$

$0.0-160.0^{\circ}$

$150.0 \mathrm{sec} . /^{\circ}$

$127.40 \mathrm{~mm}$

$0.100 \mathrm{~mm}$

$54.9^{\circ}$

Total: 9385

Unique: $2401\left(\mathrm{R}_{\mathrm{int}}=0.033\right)$

Lorentz-polarization 


\section{Structure Solution and Refinement}

Structure Solution

Refinement

Function Minimized

Least Squares Weights

Anomalous Dispersion

No. Observations $(\mathrm{I}>3.00 \sigma(\mathrm{I}))$

No. Variables

Reflection/Parameter Ratio

Residuals: R (I>3.00б(I))

Residuals: $\mathrm{Rw}(\mathrm{I}>3.00 \sigma(\mathrm{I}))$

Goodness of Fit Indicator

Max Shift/Error in Final Cycle

Maximum peak in Final Diff. Map

Minimum peak in Final Diff. Map
Direct Methods (SIR92)

Full-matrix least-squares on $\mathrm{F}$

$\Sigma_{\mathrm{w}}\left(|\mathrm{Fo}|-\left|\mathrm{FC}_{\mathrm{C}}\right|\right)^{2}$

1

All non-hydrogen atoms

2009

224

8.97

0.038

0.063

0.88

0.00

$3.01 \mathrm{e}^{-} / \AA^{3}$

$-0.43 \mathrm{e}^{-} / \AA^{3}$ 
Table 1. Atomic coordinates and $\mathrm{Biso} / \mathrm{Beq}$

$\begin{array}{lcccl}\text { atom } & \mathrm{x} & \mathrm{y} & \mathrm{z} & \mathrm{B} \mathrm{eq} \\ \mathrm{Rh}(1) & -0.05482(3) & -0.0271(8) & -0.06182(6) & 1.17(1) \\ \mathrm{Cl}(1) & -0.01118(9) & -0.1858(7) & 0.1236(2) & 1.55(5) \\ \mathrm{C}(4) & -0.1029(4) & 0.051(2) & -0.2257(9) & 1.5(2) \\ \mathrm{C}(1) & -0.1012(4) & 0.209(2) & -0.0090(9) & 1.4(2) \\ \mathrm{C}(2) & -0.1275(3) & 0.010(2) & -0.0577(8) & 1.3(2) \\ \mathrm{C}(7) & -0.1592(4) & 0.326(2) & -0.187(1) & 1.8(2) \\ \mathrm{C}(11) & -0.2150(4) & 0.511(3) & 0.1121(9) & 2.1(3) \\ \mathrm{C}(9) & -0.1288(4) & 0.432(2) & 0.1377(9) & 1.3(2) \\ \mathrm{C}(14) & -0.1179(4) & 0.639(2) & 0.2007(9) & 1.7(2) \\ \mathrm{C}(16) & -0.1401(4) & -0.005(3) & -0.4352(8) & 1.5(2) \\ \mathrm{C}(13) & -0.1546(4) & 0.779(2) & 0.220(1) & 2.1(2) \\ \mathrm{C}(21) & -0.1826(4) & -0.137(2) & -0.4763(9) & 2.0(2) \\ \mathrm{C}(20) & -0.2208(4) & -0.061(3) & -0.5698(9) & 2.2(3) \\ \mathrm{C}(19) & -0.2164(5) & 0.149(3) & -0.623(1) & 2.5(3) \\ \mathrm{C}(6) & -0.1064(4) & 0.380(2) & -0.1099(9) & 1.5(2) \\ \mathrm{C}(10) & -0.1789(4) & 0.369(2) & 0.0950(9) & 1.9(2) \\ \mathrm{C}(5) & -0.0765(4) & 0.252(2) & -0.1761(9) & 1.4(2) \\ \mathrm{C}(15) & -0.1000(4) & -0.087(2) & -0.3272(9) & 1.8(2) \\ \mathrm{C}(8) & -0.0887(4) & 0.283(2) & 0.1144(9) & 1.5(2) \\ \mathrm{C}(18) & -0.1729(5) & 0.279(2) & -0.584(1) & 2.6(3) \\ \mathrm{C}(3) & -0.1502(4) & 0.061(2) & -0.1888(9) & 1.5(2) \\ \mathrm{C}(12) & -0.2031(4) & 0.715(2) & 0.174(1) & 2.2(2) \\ \mathrm{C}(17) & -0.1362(5) & 0.201(2) & -0.490(1) & 2.4(2) \\ \mathrm{H}(7) & -0.0847(4) & 0.147(2) & 0.1609(9) & 1.7(2) \\ \mathrm{H}(8) & -0.0590(4) & 0.369(2) & 0.1340(9) & 1.7(2) \\ \mathrm{H}(15) & -0.0687(4) & -0.068(2) & -0.3383(9) & 2.1(3) \\ \mathrm{H}(14) & -0.1052(4) & -0.247(2) & -0.3141(9) & 2.1(3) \\ \mathrm{H}(2) & -0.1769(4) & -0.034(2) & -0.2303(9) & 1.9(2) \\ \mathrm{H}(4) & -0.0982(4) & 0.538(2) & -0.0911(9) & 1.8(2) \\ \mathrm{H}(18) & -0.2425(5) & 0.202(3) & -0.686(1) & 2.9(3) \\ \mathrm{H}(19) & -0.2501(4) & -0.150(3) & -0.5961(9) & 2.6(4) \\ \mathrm{H}(5) & -0.1837(4) & 0.366(2) & -0.152(1) & 2.2(2) \\ \mathrm{H}(6) & -0.1668(4) & 0.396(2) & -0.261(1) & 2.2(2) \\ \mathrm{H}(13) & -0.0846(4) & 0.685(2) & 0.2311(9) & 2.1(2) \\ \mathrm{H}(12) & -0.1465(4) & 0.918(2) & 0.265(1) & 2.7(3) \\ \mathrm{H}(11) & -0.2283(4) & 0.811(2) & 0.186(1) & 2.7(3) \\ \mathrm{H}(20) & -0.1859(4) & -0.280(2) & -0.4403(9) & 2.4(3) \\ \mathrm{H}(16) & -0.1074(5) & 0.292(2) & -0.461(1) & 2.8(3) \\ \mathrm{H}(17) & -0.1686(5) & 0.418(2) & -0.622(1) & 3.2(3) \\ \mathrm{H}(10) & -0.2484(4) & 0.469(3) & 0.0802(9) & 2.5(4) \\ \mathrm{H}(9) & -0.1877(4) & 0.227(2) & 0.0541(9) & 2.2(2) \\ \mathrm{H}(3) & -0.0545(4) & 0.330(2) & -0.2090(9) & 1.7(2) \\ \mathrm{H}(1) & -0.1423(3) & -0.092(2) & -0.0161(8) & 1.6(3)\end{array}$

$B_{\text {eq }}=8 / 3 \pi^{2}\left(U_{11}\left(a^{*}\right)^{2}+U_{22}\left(b^{*}\right)^{2}+U_{33}\left(c c^{*}\right)^{2}+2 U_{12}\left(a a^{*} b b^{*}\right) \cos \gamma+2 U_{13}\left(a a^{*} c c^{*}\right) \cos \beta+2 U_{23}\left(b b^{*} c c^{*}\right) \cos \alpha\right)$ 
Table 2. Anisotropic Displacement Parameters

$\begin{array}{lllllll}\text { atom } & \mathrm{U} 11 & \mathrm{U}_{22} & \mathrm{U} 33 & \mathrm{U} 12 & \mathrm{U} 13 & \mathrm{U} 23 \\ \mathrm{Rh}(1) & 0.0124(3) & 0.0144(3) & 0.0171(3) & 0.0000(6) & 0.0039(2) & -0.0008(6) \\ \mathrm{Cl}(1) & 0.014(1) & 0.022(1) & 0.022(1) & -0.000(1) & 0.0046(9) & 0.005(1) \\ \mathrm{C}(4) & 0.014(4) & 0.021(5) & 0.021(5) & 0.004(4) & 0.003(4) & 0.002(4) \\ \mathrm{C}(1) & 0.013(4) & 0.019(5) & 0.022(5) & 0.002(4) & 0.005(4) & -0.001(5) \\ \mathrm{C}(2) & 0.013(4) & 0.017(9) & 0.020(4) & 0.003(4) & 0.005(3) & 0.005(5) \\ \mathrm{C}(11) & 0.022(5) & 0.04(1) & 0.022(5) & 0.009(6) & 0.006(4) & 0.002(6) \\ \mathrm{C}(9) & 0.018(4) & 0.012(8) & 0.020(4) & -0.001(4) & 0.006(4) & 0.001(4) \\ \mathrm{C}(14) & 0.021(5) & 0.021(6) & 0.024(5) & -0.003(5) & 0.006(4) & -0.000(5) \\ \mathrm{C}(16) & 0.022(4) & 0.019(7) & 0.016(4) & 0.001(6) & 0.008(3) & -0.003(6) \\ \mathrm{C}(13) & 0.038(6) & 0.022(6) & 0.024(6) & 0.002(5) & 0.015(5) & -0.003(5) \\ \mathrm{C}(21) & 0.029(6) & 0.026(6) & 0.023(5) & 0.004(5) & 0.009(5) & 0.000(5) \\ \mathrm{C}(20) & 0.020(5) & 0.04(1) & 0.023(5) & 0.000(6) & 0.003(4) & -0.001(6) \\ \mathrm{C}(19) & 0.030(6) & 0.041(8) & 0.020(5) & 0.016(6) & 0.004(5) & 0.001(6) \\ \mathrm{C}(6) & 0.019(5) & 0.015(5) & 0.023(5) & 0.002(4) & 0.007(4) & 0.000(4) \\ \mathrm{C}(10) & 0.019(5) & 0.030(6) & 0.021(5) & -0.001(5) & 0.005(4) & -0.004(5) \\ \mathrm{C}(5) & 0.015(5) & 0.020(6) & 0.020(5) & 0.000(4) & 0.005(4) & 0.004(4) \\ \mathrm{C}(15) & 0.018(5) & 0.027(8) & 0.024(5) & 0.002(4) & 0.005(4) & -0.002(4) \\ \mathrm{C}(8) & 0.014(4) & 0.020(6) & 0.021(5) & 0.000(4) & 0.003(4) & -0.001(5) \\ \mathrm{C}(18) & 0.055(8) & 0.024(7) & 0.024(6) & 0.008(6) & 0.015(6) & 0.003(5) \\ \mathrm{C}(3) & 0.016(5) & 0.020(5) & 0.024(5) & -0.004(4) & 0.007(4) & -0.003(4) \\ \mathrm{C}(12) & 0.028(6) & 0.035(7) & 0.023(5) & 0.014(6) & 0.012(5) & 0.002(5) \\ \mathrm{C}(17) & 0.031(6) & 0.028(7) & 0.030(6) & -0.003(6) & 0.007(5) & -0.001(6)\end{array}$

The general temperature factor expression: $\exp \left(-2 \pi^{2}\left(a^{* 2} U_{11} h^{2}+b^{* 2} U_{22} k^{2}+c^{* 2} U_{33}{ }^{2}\right.\right.$ $\left.\left.+2 a^{*} b^{*} U_{12} h k+2 a^{*} c^{*} U_{13} h l+2 b^{*} c^{*} U_{23} k l\right)\right)$ 
Table 3. Bond lengths ( $\mathrm{A})$

$\begin{array}{llllll}\text { atom } & \text { atom } & \text { distance } & \text { atom } & \text { atom } & \begin{array}{l}\text { distance } \\ \operatorname{Rh}(1)\end{array} \\ \mathrm{Cl}(1) & 2.410(3) & \mathrm{Rh}(1) & \mathrm{C}(4) & 2.11(1) \\ \operatorname{Rh}(1) & \mathrm{C}(1) & 2.12(1) & \mathrm{Rh}(1) & \mathrm{C}(2) & 2.101(9) \\ \operatorname{Rh}(1) & \mathrm{C}(5) & 2.09(1) & \mathrm{C}(4) & \mathrm{C}(5) & 1.41(2) \\ \mathrm{C}(4) & \mathrm{C}(15) & 1.49(2) & \mathrm{C}(4) & \mathrm{C}(3) & 1.54(1) \\ \mathrm{C}(1) & \mathrm{C}(2) & 1.40(2) & \mathrm{C}(1) & \mathrm{C}(6) & 1.54(2) \\ \mathrm{C}(1) & \mathrm{C}(8) & 1.50(1) & \mathrm{C}(2) & \mathrm{C}(3) & 1.56(1) \\ \mathrm{C}(7) & \mathrm{C}(6) & 1.55(1) & \mathrm{C}(7) & \mathrm{C}(3) & 1.54(2) \\ \mathrm{C}(11) & \mathrm{C}(10) & 1.38(2) & \mathrm{C}(11) & \mathrm{C}(12) & 1.38(2) \\ \mathrm{C}(9) & \mathrm{C}(14) & 1.40(2) & \mathrm{C}(9) & \mathrm{C}(10) & 1.42(1) \\ \mathrm{C}(9) & \mathrm{C}(8) & 1.52(1) & \mathrm{C}(14) & \mathrm{C}(13) & 1.40(2) \\ \mathrm{C}(16) & \mathrm{C}(21) & 1.39(2) & \mathrm{C}(16) & \mathrm{C}(15) & 1.54(1) \\ \mathrm{C}(16) & \mathrm{C}(17) & 1.38(2) & \mathrm{C}(13) & \mathrm{C}(12) & 1.38(2) \\ \mathrm{C}(21) & \mathrm{C}(20) & 1.39(2) & \mathrm{C}(20) & \mathrm{C}(19) & 1.39(2) \\ \mathrm{C}(19) & \mathrm{C}(18) & 1.41(2) & \mathrm{C}(6) & \mathrm{C}(5) & 1.52(1) \\ \mathrm{C}(18) & \mathrm{C}(17) & 1.38(2) & & & \end{array}$

Table 4. Bond lengths involving hydrogens ( $\mathrm{A})$

$\begin{array}{llllll}\text { atom } & \text { atom } & \text { distance } & \text { atom } & \text { atom } & \text { distance } \\ \mathrm{Rh}(1) & \mathrm{Cl}(1) & 2.410(3) & \mathrm{Rh}(1) & \mathrm{C}(4) & 2.11(1) \\ \mathrm{Rh}(1) & \mathrm{C}(1) & 2.12(1) & \mathrm{Rh}(1) & \mathrm{C}(2) & 2.101(9) \\ \mathrm{Rh}(1) & \mathrm{C}(5) & 2.09(1) & \mathrm{C}(2) & \mathrm{H}(1) & 0.95(2) \\ \mathrm{C}(7) & \mathrm{H}(5) & 0.95(2) & \mathrm{C}(7) & \mathrm{H}(6) & 0.95(2) \\ \mathrm{C}(11) & \mathrm{H}(10) & 0.95(2) & \mathrm{C}(14) & \mathrm{H}(13) & 0.95(2) \\ \mathrm{C}(13) & \mathrm{H}(12) & 0.95(2) & \mathrm{C}(21) & \mathrm{H}(20) & 0.95(2) \\ \mathrm{C}(20) & \mathrm{H}(19) & 0.95(2) & \mathrm{C}(19) & \mathrm{H}(18) & 0.95(2) \\ \mathrm{C}(6) & \mathrm{H}(4) & 0.95(2) & \mathrm{C}(10) & \mathrm{H}(9) & 0.95(2) \\ \mathrm{C}(5) & \mathrm{H}(3) & 0.95(2) & \mathrm{C}(15) & \mathrm{H}(15) & 0.95(2) \\ \mathrm{C}(15) & \mathrm{H}(14) & 0.95(2) & \mathrm{C}(8) & \mathrm{H}(7) & 0.95(2) \\ \mathrm{C}(8) & \mathrm{H}(8) & 0.95(1) & \mathrm{C}(18) & \mathrm{H}(17) & 0.95(2) \\ \mathrm{C}(3) & \mathrm{H}(2) & 0.95(2) & \mathrm{C}(12) & \mathrm{H}(11) & 0.95(2) \\ \mathrm{C}(17) & \mathrm{H}(16) & 0.95(2) & & & \end{array}$


Table 5. Bond angles ( $\left.{ }^{\mathrm{O}}\right)$

$\begin{array}{llllllll}\text { atom } & \text { atom } & \text { atom } & \text { angle } & \text { atom } & \text { atom } & \text { atom } & \text { angle } \\ \mathrm{Cl}(1) & \mathrm{Rh}(1) & \mathrm{C}(4) & 167.9(4) & \mathrm{Cl}(1) & \mathrm{Rh}(1) & \mathrm{C}(1) & 98.9(3) \\ \mathrm{C}(4) & \mathrm{Rh}(1) & \mathrm{C}(1) & 81.9(4) & \mathrm{Cl}(1) & \mathrm{Rh}(1) & \mathrm{C}(2) & 104.9(3) \\ \mathrm{C}(4) & \mathrm{Rh}(1) & \mathrm{C}(2) & 68.2(4) & \mathrm{C}(1) & \mathrm{Rh}(1) & \mathrm{C}(2) & 38.7(4) \\ \mathrm{Cl}(1) & \mathrm{Rh}(1) & \mathrm{C}(5) & 151.8(3) & \mathrm{C}(4) & \mathrm{Rh}(1) & \mathrm{C}(5) & 39.2(4) \\ \mathrm{C}(1) & \mathrm{Rh}(1) & \mathrm{C}(5) & 67.9(4) & \mathrm{C}(2) & \mathrm{Rh}(1) & \mathrm{C}(5) & 80.7(4) \\ \mathrm{Rh}(1) & \mathrm{C}(4) & \mathrm{C}(5) & 69.7(6) & \mathrm{Rh}(1) & \mathrm{C}(4) & \mathrm{C}(15) & 119.4(7) \\ \mathrm{C}(5) & \mathrm{C}(4) & \mathrm{C}(15) & 129.5(1) & \mathrm{Rh}(1) & \mathrm{C}(4) & \mathrm{C}(3) & 96.5(7) \\ \mathrm{C}(5) & \mathrm{C}(4) & \mathrm{C}(3) & 104.5(9) & \mathrm{C}(15) & \mathrm{C}(4) & \mathrm{C}(3) & 121.7(9) \\ \mathrm{Rh}(1) & \mathrm{C}(1) & \mathrm{C}(2) & 69.8(6) & \mathrm{Rh}(1) & \mathrm{C}(1) & \mathrm{C}(6) & 94.9(6) \\ \mathrm{C}(2) & \mathrm{C}(1) & \mathrm{C}(6) & 105.5(9) & \mathrm{Rh}(1) & \mathrm{C}(1) & \mathrm{C}(8) & 119.8(7) \\ \mathrm{C}(2) & \mathrm{C}(1) & \mathrm{C}(8) & 126.4(1) & \mathrm{C}(6) & \mathrm{C}(1) & \mathrm{C}(8) & 124.0(1) \\ \mathrm{Rh}(1) & \mathrm{C}(2) & \mathrm{C}(1) & 71.5(6) & \mathrm{Rh}(1) & \mathrm{C}(2) & \mathrm{C}(3) & 96.3(6) \\ \mathrm{C}(1) & \mathrm{C}(2) & \mathrm{C}(3) & 106.8(1) & \mathrm{C}(6) & \mathrm{C}(7) & \mathrm{C}(3) & 93.8(9) \\ \mathrm{C}(10) & \mathrm{C}(11) & \mathrm{C}(12) & 120.6(1) & \mathrm{C}(14) & \mathrm{C}(9) & \mathrm{C}(10) & 117.0(1) \\ \mathrm{C}(14) & \mathrm{C}(9) & \mathrm{C}(8) & 121.5(9) & \mathrm{C}(10) & \mathrm{C}(9) & \mathrm{C}(8) & 121.5(9) \\ \mathrm{C}(9) & \mathrm{C}(14) & \mathrm{C}(13) & 121.6(1) & \mathrm{C}(21) & \mathrm{C}(16) & \mathrm{C}(15) & 119.5(1) \\ \mathrm{C}(21) & \mathrm{C}(16) & \mathrm{C}(17) & 118.6(1) & \mathrm{C}(15) & \mathrm{C}(16) & \mathrm{C}(17) & 121.8(1) \\ \mathrm{C}(14) & \mathrm{C}(13) & \mathrm{C}(12) & 119.6(1) & \mathrm{C}(16) & \mathrm{C}(21) & \mathrm{C}(20) & 121.0(1) \\ \mathrm{C}(21) & \mathrm{C}(20) & \mathrm{C}(19) & 119.6(1) & \mathrm{C}(20) & \mathrm{C}(19) & \mathrm{C}(18) & 119.5(1) \\ \mathrm{C}(1) & \mathrm{C}(6) & \mathrm{C}(7) & 101.7(9) & \mathrm{C}(1) & \mathrm{C}(6) & \mathrm{C}(5) & 100.4(9) \\ \mathrm{C}(7) & \mathrm{C}(6) & \mathrm{C}(5) & 100.6(8) & \mathrm{C}(11) & \mathrm{C}(10) & \mathrm{C}(9) & 121.1(1) \\ \operatorname{Rh}(1) & \mathrm{C}(5) & \mathrm{C}(4) & 71.1(6) & \mathrm{Rh}(1) & \mathrm{C}(5) & \mathrm{C}(6) & 96.8(7) \\ \mathrm{C}(4) & \mathrm{C}(5) & \mathrm{C}(6) & 107.8(9) & \mathrm{C}(4) & \mathrm{C}(15) & \mathrm{C}(16) & 109.7(1) \\ \mathrm{C}(1) & \mathrm{C}(8) & \mathrm{C}(9) & 112.1(8) & \mathrm{C}(19) & \mathrm{C}(18) & \mathrm{C}(17) & 119.4(1) \\ \mathrm{C}(4) & \mathrm{C}(3) & \mathrm{C}(2) & 98.9(8) & \mathrm{C}(4) & \mathrm{C}(3) & \mathrm{C}(7) & 101.7(9) \\ \mathrm{C}(2) & \mathrm{C}(3) & \mathrm{C}(7) & 100.8(9) & \mathrm{C}(11) & \mathrm{C}(12) & \mathrm{C}(13) & 120.1(1) \\ \mathrm{C}(16) & \mathrm{C}(17) & \mathrm{C}(18) & 121.9(1) & & & & \end{array}$


Table 6. Bond angles involving hydrogens $\left({ }^{\circ}\right)$

\begin{tabular}{|c|c|c|c|c|c|c|c|}
\hline $\mathrm{pm}$ & atom & atom & angle & atom & atom & atom & angle \\
\hline $\mathrm{Cl}(1)$ & $\operatorname{Rh}(1)$ & $C(4)$ & $167.9(4)$ & $\mathrm{Cl}(1)$ & $\operatorname{Rh}(1)$ & $C(1)$ & $98.9(3)$ \\
\hline$C(4)$ & $\operatorname{Rh}(1)$ & $C(1)$ & $81.9(4)$ & $\mathrm{Cl}(1)$ & $\operatorname{Rh}(1)$ & $C(2)$ & $104.9(3)$ \\
\hline C(4) & $\operatorname{Rh}(1)$ & $C(2)$ & $68.2(4)$ & $C(1)$ & $\operatorname{Rh}(1)$ & $C(2)$ & $38.7(4)$ \\
\hline $\mathrm{Cl}(1)$ & $\operatorname{Rh}(1)$ & $C(5)$ & $151.8(3)$ & $C(4)$ & $\operatorname{Rh}(1)$ & $C(5)$ & $39.2(4)$ \\
\hline$C(1)$ & $\operatorname{Rh}(1)$ & $C(5)$ & $67.9(4)$ & $C(2)$ & $\operatorname{Rh}(1)$ & $C(5)$ & $80.7(4)$ \\
\hline $\operatorname{Rh}(1)$ & $C(4)$ & $C(5)$ & $69.7(6)$ & $\operatorname{Rh}(1)$ & $C(4)$ & $C(15)$ & $119.4(7)$ \\
\hline $\operatorname{Rh}(1)$ & $C(4)$ & $C(3)$ & $96.5(7)$ & $\operatorname{Rh}(1)$ & $C(1)$ & $C(2)$ & $69.8(6)$ \\
\hline $\operatorname{Rh}(1)$ & $C(1)$ & $C(6)$ & $94.9(6)$ & $\operatorname{Rh}(1)$ & $C(1)$ & $C(8)$ & $119.8(7)$ \\
\hline $\operatorname{Rh}(1)$ & $C(2)$ & $C(1)$ & $71.5(6)$ & $\operatorname{Rh}(1)$ & $C(2)$ & $C(3)$ & $96.3(6)$ \\
\hline $\operatorname{Rh}(1)$ & $C(2)$ & $\mathrm{H}(1)$ & $123.2(1)$ & $C(1)$ & $C(2)$ & $\mathrm{H}(1)$ & $123.1(1)$ \\
\hline$C(3)$ & $C(2)$ & $\mathrm{H}(1)$ & $122.9(1)$ & $C(6)$ & $C(7)$ & $\mathrm{H}(5)$ & $113.3(1)$ \\
\hline$C(3)$ & $C(7)$ & $\mathrm{H}(5)$ & $113.2(1)$ & $C(6)$ & $C(7)$ & $\mathrm{H}(6)$ & $113.4(1)$ \\
\hline$C(3)$ & $C(7)$ & $\mathrm{H}(6)$ & $113.0(1)$ & $\mathrm{H}(5)$ & $C(7)$ & $\mathrm{H}(6)$ & $109.5(2)$ \\
\hline$C(10)$ & C(11) & $\mathrm{H}(10)$ & $119.9(2)$ & $C(12)$ & $C(11)$ & $\mathrm{H}(10)$ & $119.5(2)$ \\
\hline$C(9)$ & C(14) & $\mathrm{H}(13)$ & $119.0(1)$ & $C(13)$ & C(14) & $\mathrm{H}(13)$ & $119.4(1)$ \\
\hline$C(14)$ & C(13) & $\mathrm{H}(12)$ & $120.5(2)$ & $C(12)$ & $C(13)$ & $\mathrm{H}(12)$ & $119.9(2)$ \\
\hline$C(16)$ & $C(21)$ & $\mathrm{H}(20)$ & $119.9(2)$ & $C(20)$ & $C(21)$ & $\mathrm{H}(20)$ & $119.0(2)$ \\
\hline$C(21)$ & $C(20)$ & $\mathrm{H}(19)$ & $120.6(2)$ & $C(19)$ & $C(20)$ & $\mathrm{H}(19)$ & $119.9(2)$ \\
\hline$C(20)$ & C(19) & $\mathrm{H}(18)$ & $119.8(2)$ & $C(18)$ & C(19) & $\mathrm{H}(18)$ & $120.8(2)$ \\
\hline$C(1)$ & $C(6)$ & $\mathrm{H}(4)$ & 117.1(1) & $C(7)$ & $C(6)$ & $\mathrm{H}(4)$ & $117.3(1)$ \\
\hline$C(5)$ & $C(6)$ & $\mathrm{H}(4)$ & $116.8(1)$ & $C(11)$ & $C(10)$ & $\mathrm{H}(9)$ & $119.5(1)$ \\
\hline$C(9)$ & $C(10)$ & $\mathrm{H}(9)$ & $119.4(1)$ & $\operatorname{Rh}(1)$ & $C(5)$ & $C(4)$ & $71.1(6)$ \\
\hline $\operatorname{Rh}(1)$ & $C(5)$ & $C(6)$ & $96.8(7)$ & $\operatorname{Rh}(1)$ & C(5) & $\mathrm{H}(3)$ & $122.5(1)$ \\
\hline$C(4)$ & $C(5)$ & $\mathrm{H}(3)$ & $122.7(1)$ & $C(6)$ & $C(5)$ & $\mathrm{H}(3)$ & $122.7(1)$ \\
\hline$C(4)$ & $C(15)$ & $\mathrm{H}(15)$ & $110.1(1)$ & $C(16)$ & $C(15)$ & $\mathrm{H}(15)$ & $109.8(1)$ \\
\hline$C(4)$ & $C(15)$ & $\mathrm{H}(14)$ & $108.9(1)$ & $C(16)$ & $C(15)$ & $\mathrm{H}(14)$ & $108.9(1)$ \\
\hline $\mathrm{H}(15)$ & $C(15)$ & $\mathrm{H}(14)$ & $109.5(1)$ & $\mathrm{C}(1)$ & $C(8)$ & $\mathrm{H}(7)$ & 108.5(1) \\
\hline C(9) & $C(8)$ & $\mathrm{H}(7)$ & $108.5(1)$ & $\mathrm{C}(1)$ & $C(8)$ & $\mathrm{H}(8)$ & $109.2(1)$ \\
\hline$C(9)$ & $C(8)$ & $\mathrm{H}(8)$ & $109.0(1)$ & $\mathrm{H}(7)$ & $C(8)$ & $\mathrm{H}(8)$ & 109.5(1) \\
\hline$C(19)$ & C(18) & $\mathrm{H}(17)$ & $120.5(2)$ & $C(17)$ & $C(18)$ & $\mathrm{H}(17)$ & $120.1(2)$ \\
\hline$C(4)$ & $C(3)$ & $\mathrm{H}(2)$ & $117.3(1)$ & $C(2)$ & $C(3)$ & $\mathrm{H}(2)$ & $117.6(1)$ \\
\hline$C(7)$ & $C(3)$ & $\mathrm{H}(2)$ & $117.4(1)$ & C(11) & $C(12)$ & $\mathrm{H}(11)$ & $119.9(2)$ \\
\hline C(13) & $C(12)$ & $\mathrm{H}(11)$ & $120.0(2)$ & $C(16)$ & C(17) & $\mathrm{H}(16)$ & $118.7(2)$ \\
\hline$C(18)$ & $C(17)$ & $\mathrm{H}(16)$ & $119.4(2)$ & & & & \\
\hline
\end{tabular}


Table 7. Torsion Angles(O)

\begin{tabular}{|c|c|c|c|c|c|c|c|c|c|}
\hline atom & H & & HII & angle & atom & atom & atom & atom & angle \\
\hline $\mathrm{Rh}(1)$ & $C(4)$ & $C(5)$ & $\operatorname{Rh}(1)$ & $0.0(2)$ & $\operatorname{Rh}(1)$ & $C(4)$ & $C(5)$ & $C(6)$ & $-91.2(1)$ \\
\hline$C(15)$ & $C(4)$ & $C(5)$ & $\operatorname{Rh}(1)$ & $-111.8(2)$ & $C(15)$ & $C(4)$ & $C(5)$ & $C(6)$ & $156.9(2)$ \\
\hline$C(3)$ & $C(4)$ & $C(5)$ & $\operatorname{Rh}(1)$ & 91.7(1) & $C(3)$ & $C(4)$ & $C(5)$ & $C(6)$ & $0.5(2)$ \\
\hline $\mathrm{Rh}(1)$ & $C(4)$ & $C(15)$ & $C(16)$ & $177.3(9)$ & $C(5)$ & $C(4)$ & $C(15)$ & $C(16)$ & $-95.7(2)$ \\
\hline$C(3)$ & $C(4)$ & $\mathrm{C}(15)$ & $C(16)$ & $57.2(2)$ & $\operatorname{Rh}(1)$ & $C(4)$ & $C(3)$ & $C(2)$ & $1.0(1)$ \\
\hline $\mathrm{Rh}(1)$ & $C(4)$ & $C(3)$ & $C(7)$ & $104.1(1)$ & $C(5)$ & $C(4)$ & $C(3)$ & $C(2)$ & $-69.7(2)$ \\
\hline$C(5)$ & $C(4)$ & $C(3)$ & $C(7)$ & $33.4(2)$ & $C(15)$ & $C(4)$ & $C(3)$ & $C(2)$ & $131.6(2)$ \\
\hline$C(15)$ & $C(4)$ & $C(3)$ & $C(7)$ & $-125.3(2)$ & $\operatorname{Rh}(1)$ & $C(1)$ & $C(2)$ & $\operatorname{Rh}(1)$ & $0.0(2)$ \\
\hline $\mathrm{Rh}(1)$ & $C(1)$ & $C(2)$ & $C(3)$ & $-91.2(1)$ & $C(6)$ & $C(1)$ & $C(2)$ & $\operatorname{Rh}(1)$ & $89.6(1)$ \\
\hline$C(6)$ & $\mathrm{C}(1)$ & $C(2)$ & $C(3)$ & $-1.6(2)$ & $C(8)$ & $C(1)$ & $C(2)$ & $\operatorname{Rh}(1)$ & $-112.8(2)$ \\
\hline $\mathrm{C}(8)$ & $\mathrm{C}(1)$ & $C(2)$ & $C(3)$ & $156.0(1)$ & $\operatorname{Rh}(1)$ & $\mathrm{C}(1)$ & $C(6)$ & $C(7)$ & 105.3(1) \\
\hline $\mathrm{Rh}(1)$ & $C(1)$ & $C(6)$ & $C(5)$ & 2.1(1) & $C(2)$ & $C(1)$ & $C(6)$ & $C(7)$ & $34.9(2)$ \\
\hline$C(2)$ & $C(1)$ & $C(6)$ & $C(5)$ & $-68.3(2)$ & $C(8)$ & $C(1)$ & $C(6)$ & $C(7)$ & $-123.4(2)$ \\
\hline $\mathrm{C}(8)$ & $C(1)$ & $C(6)$ & $C(5)$ & $133.4(2)$ & $\operatorname{Rh}(1)$ & $C(1)$ & $C(8)$ & $C(9)$ & $-174.2(8)$ \\
\hline$C(2)$ & $\mathrm{C}(1)$ & $C(8)$ & $C(9)$ & $-88.2(2)$ & $C(6)$ & $C(1)$ & $C(8)$ & $C(9)$ & $65.5(2)$ \\
\hline $\mathrm{Rh}(1)$ & $C(2)$ & $C(3)$ & $C(4)$ & $-1.0(1)$ & $\operatorname{Rh}(1)$ & $C(2)$ & $C(3)$ & $C(7)$ & $-104.8(1)$ \\
\hline$C(1)$ & $C(2)$ & $C(3)$ & $C(4)$ & $71.5(2)$ & $C(1)$ & $C(2)$ & $C(3)$ & $C(7)$ & $-32.3(2)$ \\
\hline$C(3)$ & $C(7)$ & $C(6)$ & $C(1)$ & $-52.0(1)$ & $C(3)$ & $\mathrm{C}(7)$ & $C(6)$ & $C(5)$ & 51.1(1) \\
\hline C(6) & $\mathrm{C}(7)$ & $C(3)$ & $C(4)$ & $-51.4(1)$ & $C(6)$ & $\mathrm{C}(7)$ & $C(3)$ & $C(2)$ & $50.2(1)$ \\
\hline$C(12)$ & $C(11)$ & $C(10)$ & $C(9)$ & $-1.9(3)$ & $C(10)$ & $C(11)$ & $C(12)$ & $C(13)$ & $-0.1(3)$ \\
\hline$C(10)$ & C(9) & $C(14)$ & $C(13)$ & $-0.3(2)$ & $C(8)$ & $C(9)$ & $C(14)$ & $C(13)$ & $178.7(1)$ \\
\hline$C(14)$ & $C(9)$ & $C(10)$ & $C(11)$ & $2.1(2)$ & $C(8)$ & $C(9)$ & $C(10)$ & $C(11)$ & $-176.9(1)$ \\
\hline$C(14)$ & C(9) & $\mathrm{C}(8)$ & $\mathrm{C}(1)$ & $-133.2(2)$ & $\mathrm{C}(10)$ & $C(9)$ & $C(8)$ & $\mathrm{C}(1)$ & $45.8(2)$ \\
\hline$C(9)$ & $C(14)$ & $C(13)$ & $C(12)$ & $-1.6(2)$ & $C(15)$ & $C(16)$ & $C(21)$ & $C(20)$ & 176.3(1) \\
\hline$C(17)$ & $C(16)$ & $C(21)$ & $C(20)$ & $-0.7(3)$ & $\mathrm{C}(21)$ & $C(16)$ & $C(15)$ & $C(4)$ & $-101.6(2)$ \\
\hline$C(17)$ & $C(16)$ & $C(15)$ & $C(4)$ & $75.3(2)$ & $C(21)$ & $C(16)$ & $C(17)$ & $C(18)$ & $-0.7(3)$ \\
\hline$C(15)$ & $C(16)$ & $C(17)$ & $C(18)$ & $-177.6(2)$ & $\mathrm{C}(14)$ & $C(13)$ & $C(12)$ & $C(11)$ & $1.8(3)$ \\
\hline$C(16)$ & $\mathrm{C}(21)$ & $\mathrm{C}(20)$ & C(19) & $0.2(3)$ & $\mathrm{C}(21)$ & $C(20)$ & C(19) & $C(18)$ & $1.7(3)$ \\
\hline$C(20)$ & $C(19)$ & $C(18)$ & $C(17)$ & $-3.0(3)$ & $\mathrm{C}(1)$ & $C(6)$ & $C(5)$ & $\operatorname{Rh}(1)$ & $-2.1(1)$ \\
\hline$C(1)$ & $C(6)$ & $\mathrm{C}(5)$ & $C(4)$ & $70.2(2)$ & $C(7)$ & $C(6)$ & $C(5)$ & $\operatorname{Rh}(1)$ & $-106.3(1$ \\
\hline$C(7)$ & $\mathrm{C}(6)$ & $\mathrm{C}(5)$ & $C(4)$ & $-34.0(2)$ & C(19) & $C(18)$ & $\mathrm{C}(17)$ & $C(16)$ & $2.5(3)$ \\
\hline
\end{tabular}


Table 8. Distances beyond the asymmetric unit out to $3.60 \AA$

\begin{tabular}{|c|c|c|c|c|c|c|c|}
\hline atom & atom & distance & ADC & atom & atom & distance & ADC \\
\hline $\mathrm{RH}(1)$ & $\mathrm{CL}(1)$ & $2.41(5)$ & (1) & $\mathrm{RH}(1)$ & $\mathrm{CL}(1)$ & $2.40(5)$ & (2) \\
\hline $\mathrm{RH}(1)$ & $C(4)$ & $2.11(5)$ & (1) & $\mathrm{RH}(1)$ & $\mathrm{C}(1)$ & $2.12(3)$ & (1) \\
\hline RH(1) & $C(2)$ & $2.10(2)$ & (1) & $\mathrm{RH}(1)$ & $C(7)$ & 3.55(9) & (1) \\
\hline $\mathrm{RH}(1)$ & $C(6)$ & $2.73(4)$ & (1) & $\mathrm{RH}(1)$ & $C(5)$ & 2.09(3) & (1) \\
\hline RH(1) & $C(15)$ & $3.12(8)$ & (1) & $\mathrm{RH}(1)$ & $C(8)$ & $3.15(5)$ & (1) \\
\hline RH(1) & $C(3)$ & $2.75(8)$ & (1) & CL(1) & CL(1) & $3.253(5)$ & (2) \\
\hline CL(1) & $C(4)$ & 3.41(1) & (2) & CL(1) & $C(1)$ & $3.45(1)$ & (1) \\
\hline CL(1) & $C(2)$ & 3.58(1) & (1) & CL(1) & $C(14)$ & 3.58(1) & $(1,1,0,-1)$ \\
\hline CL(1) & $C(5)$ & $3.47(1)$ & (2) & CL(1) & $C(15)$ & $3.45(1)$ & (2) \\
\hline CL(1) & C(8) & $3.46(1)$ & (1) & $C(4)$ & $C(1)$ & $2.77(2)$ & (1) \\
\hline$C(4)^{\prime}$ & $C(2)$ & $2.36(1)$ & (1) & $C(4)$ & $C(7)$ & $2.40(2)$ & (1) \\
\hline$C(4)$ & $C(16)$ & $2.48(1)$ & (1) & $C(4)$ & $\mathrm{C}(21)$ & $3.40(2)$ & (1) \\
\hline$C(4)$ & $C(6)$ & $2.37(2)$ & (1) & $C(4)$ & $C(5)$ & 1.41(2) & (1) \\
\hline$C(4)$ & $C(15)$ & $1.49(2)$ & (1) & $C(4)$ & $C(3)$ & $1.54(1)$ & (1) \\
\hline$C(4)$ & $C(17)$ & $3.19(2)$ & (1) & $C(1)$ & $C(2)$ & $1.40(2)$ & (1) \\
\hline$C(1)$ & $C(7)$ & $2.40(2)$ & (1) & $C(1)$ & $C(9)$ & $2.50(1)$ & (1) \\
\hline$C(1)$ & $C(6)$ & $1.54(2)$ & (1) & $C(1)$ & $C(10)$ & $3.00(2)$ & (1) \\
\hline$C(1)$ & $C(5)$ & $2.35(1)$ & (1) & $C(1)$ & $C(8)$ & $1.50(1)$ & (1) \\
\hline$C(1)$ & $C(3)$ & $2.38(2)$ & (1) & $C(2)$ & $C(7)$ & $2.40(2)$ & (1) \\
\hline$C(2)$ & $C(9)$ & $3.40(2)$ & (1) & $C(2)$ & $C(6)$ & $2.34(2)$ & (1) \\
\hline$C(2)$ & $C(10)$ & $3.38(2)$ & (1) & $C(2)$ & $C(5)$ & 2.71(2) & (1) \\
\hline$C(2)$ & $C(8)$ & $2.59(2)$ & (1) & $C(2)$ & $C(3)$ & $1.56(1)$ & (1) \\
\hline $\mathrm{C}(7)$ & $C(6)$ & 1.55(1) & (1) & $C(7)$ & $C(5)$ & $2.37(2)$ & (1) \\
\hline$C(7)$ & $C(3)$ & $1.54(2)$ & (1) & $C(11)$ & $C(9)$ & $2.43(2)$ & (1) \\
\hline$C(11)$ & $C(14)$ & $2.76(2)$ & (1) & $C(11)$ & $C(13)$ & $2.39(2)$ & (1) \\
\hline$C(11)$ & $C(10)$ & $1.38(2)$ & (1) & $\mathrm{C}(11)$ & $\mathrm{C}(12)$ & $1.38(2)$ & (1) \\
\hline$C(9)$ & $C(14)$ & $1.40(2)$ & (1) & $C(9)$ & $C(13)$ & $2.44(2)$ & (1) \\
\hline$C(9)$ & $C(6)$ & $3.27(1)$ & (1) & $C(9)$ & $C(10)$ & $1.42(1)$ & (1) \\
\hline$C(9)$ & C(8) & $1.52(1)$ & (1) & C(9) & $\mathrm{C}(12)$ & $2.81(2)$ & (1) \\
\hline$C(14)$ & $C(13)$ & $1.40(2)$ & (1) & $\mathrm{C}(14)$ & $\mathrm{C}(10)$ & $2.40(2)$ & (1) \\
\hline$C(14)$ & $C(8)$ & $2.54(2)$ & (1) & $C(14)$ & $C(12)$ & $2.40(2)$ & (1) \\
\hline$C(16)$ & $C(21)$ & $1.39(2)$ & (1) & $C(16)$ & $C(20)$ & $2.42(1)$ & (1) \\
\hline$C(16)$ & $C(19)$ & $2.80(2)$ & (1) & $C(16)$ & $C(5)$ & $3.47(2)$ & (1) \\
\hline$C(16)$ & $C(15)$ & $1.54(1)$ & (1) & $C(16)$ & $\mathrm{C}(18)$ & $2.41(2)$ & (1) \\
\hline$C(16)$ & $C(3)$ & $3.12(1)$ & (1) & C(16) & $\mathrm{C}(17)$ & $1.38(2)$ & (1) \\
\hline$C(13)$ & $C(10)$ & $2.77(2)$ & (1) & $C(13)$ & $C(12)$ & $1.38(2)$ & (1) \\
\hline$C(21)$ & $\mathrm{C}(20)$ & $1.39(2)$ & (1) & $\mathrm{C}(21)$ & $\mathrm{C}(19)$ & $2.41(2)$ & (1) \\
\hline
\end{tabular}


Table 8. Distances beyond the asymmetric unit out to $3.60 \AA$ (continued)

$\begin{array}{llllllll}\text { atom } & \text { atom } & \text { distance } & \text { ADC } & \text { atom } & \text { atom } & \text { distance } & \text { ADC } \\ \mathrm{C}(21) & \mathrm{C}(15) & 2.53(2) & (1) & \mathrm{C}(21) & \mathrm{C}(18) & 2.77(2) & (1) \\ \mathrm{C}(21) & \mathrm{C}(3) & 3.53(2) & (1) & \mathrm{C}(21) & \mathrm{C}(17) & 2.38(2) & (1) \\ \mathrm{C}(20) & \mathrm{C}(19) & 1.39(2) & (1) & \mathrm{C}(20) & \mathrm{C}(18) & 2.42(2) & (1) \\ \mathrm{C}(20) & \mathrm{C}(12) & 3.54(2) & (1,1,0,-1,-1) & \mathrm{C}(20) & \mathrm{C}(17) & 2.77(2) & (1) \\ \mathrm{C}(19) & \mathrm{C}(18) & 1.41(2) & (1) & \mathrm{C}(19) & \mathrm{C}(12) & 3.60(2) & (1,1,0,-1,-1) \\ \mathrm{C}(19) & \mathrm{C}(17) & 2.40(2) & (1) & \mathrm{C}(6) & \mathrm{C}(5) & 1.52(1) & (1) \\ \mathrm{C}(6) & \mathrm{C}(8) & 2.68(1) & (1) & \mathrm{C}(6) & \mathrm{C}(3) & 2.26(2) & (1) \\ \mathrm{C}(10) & \mathrm{C}(8) & 2.56(1) & (1) & \mathrm{C}(10) & \mathrm{C}(12) & 2.39(2) & (1) \\ \mathrm{C}(5) & \mathrm{C}(15) & 2.62(2) & (1) & \mathrm{C}(5) & \mathrm{C}(3) & 2.34(1) & (1) \\ \mathrm{C}(15) & \mathrm{C}(3) & 2.65(2) & (1) & \mathrm{C}(15) & \mathrm{C}(17) & 2.55(2) & (1) \\ \mathrm{C}(18) & \mathrm{C}(17) & 1.38(2) & (1) & & & & \end{array}$

*ADC (S, L, TX, TY, TZ) represents:

S: Symmetry operation number. $-\mathrm{S}$ is inversion of operation $\mathrm{S}$.
( 1) $X, Y, Z$
( 2) $-X, Y,-Z$

L: Lattice translation.

(1) $0,0,0$
( 2) $1 / 2,1 / 2,0$

TX, TY, TZ: Unit cell translation along the $\mathrm{x}, \mathrm{y}$, and $\mathrm{z}$ directions. 
Table 9. Distances beyond the asymmetric unit out to $3.60 \AA$

\begin{tabular}{|c|c|c|c|c|c|c|c|}
\hline atom & atom & distance & $\mathrm{ADC}$ & atom & atom & distance & $\mathrm{ADC}$ \\
\hline $\mathrm{RH}(1)$ & CL(1) & $2.41(5)$ & (1) & $\mathrm{RH}(1)$ & CL(1) & $2.40(5)$ & (2) \\
\hline $\mathrm{RH}(1)$ & $C(4)$ & 2.11(5) & (1) & $\mathrm{RH}(1)$ & $\mathrm{C}(1)$ & $2.12(3)$ & (1) \\
\hline $\mathrm{RH}(1)$ & $C(2)$ & $2.10(2)$ & (1) & $\mathrm{RH}(1)$ & $C(7)$ & $3.55(9)$ & (1) \\
\hline $\mathrm{RH}(1)$ & $C(6)$ & $2.73(4)$ & (1) & $\mathrm{RH}(1)$ & $C(5)$ & $2.09(3)$ & (1) \\
\hline $\mathrm{RH}(1)$ & $C(15)$ & $3.12(8)$ & (1) & $\mathrm{RH}(1)$ & $C(8)$ & $3.15(5)$ & (1) \\
\hline $\mathrm{RH}(1)$ & $C(3)$ & $2.75(8)$ & (1) & CL(1) & $\mathrm{H}(7)$ & $2.97(4)$ & (1) \\
\hline CL(1) & $\mathrm{H}(8)$ & $2.91(4)$ & $(1,1,0,-1)$ & CL(1) & $\mathrm{H}(8)$ & $3.48(4)$ & (1) \\
\hline CL(1) & $\mathrm{H}(15)$ & $3.0(2)$ & (2) & CL(1) & $\mathrm{H}(14)$ & $3.5(3)$ & (2) \\
\hline CL(1) & $\mathrm{H}(4)$ & $3.4(1)$ & $(1,1,0,-1)$ & CL(1) & $\mathrm{H}(13)$ & $2.87(9)$ & $(1,1,0,-1)$ \\
\hline CL(1) & $\mathrm{H}(3)$ & $3.3(1)$ & $(2,1,0,-1)$ & CL(1) & $\mathrm{H}(3)$ & $3.5(1)$ & (2) \\
\hline$C(4)$ & $\mathrm{H}(15)$ & $2.02(4)$ & (1) & $C(4)$ & $\mathrm{H}(14)$ & $2.01(3)$ & (1) \\
\hline$C(4)$ & $\mathrm{H}(2)$ & $2.15(3)$ & (1) & $\mathrm{C}(4)$ & $\mathrm{H}(4)$ & $3.35(5)$ & $(1,1,0,-1)$ \\
\hline$C(4)$ & $\mathrm{H}(4)$ & $3.22(5)$ & (1) & $\mathrm{C}(4)$ & $\mathrm{H}(5)$ & $3.26(7)$ & (1) \\
\hline$C(4)$ & $\mathrm{H}(6)$ & $2.64(4)$ & (1) & $\mathrm{C}(4)$ & $\mathrm{H}(20)$ & $3.5(1)$ & (1) \\
\hline$C(4)$ & $\mathrm{H}(16)$ & $3.15(6)$ & (1) & $\mathrm{C}(4)$ & $\mathrm{H}(3)$ & $2.08(3)$ & (1) \\
\hline$C(4)$ & $\mathrm{H}(1)$ & $3.18(7)$ & (1) & $\mathrm{C}(1)$ & $\mathrm{H}(7)$ & $2.01(3)$ & (1) \\
\hline$C(1)$ & $\mathrm{H}(8)$ & $2.02(5)$ & (1) & $\mathrm{C}(1)$ & $\mathrm{H}(2)$ & $3.2(1)$ & (1) \\
\hline$C(1)$ & $\mathrm{H}(4)$ & $2.15(4)$ & (1) & $C(1)$ & $\mathrm{H}(5)$ & $2.64(8)$ & (1) \\
\hline$C(1)$ & $\mathrm{H}(6)$ & $3.3(1)$ & (1) & $\mathrm{C}(1)$ & $\mathrm{H}(9)$ & $2.79(6)$ & (1) \\
\hline$C(1)$ & $\mathrm{H}(3)$ & $3.18(8)$ & (1) & $\mathrm{C}(1)$ & $\mathrm{H}(1)$ & $2.08(3)$ & (1) \\
\hline$C(2)$ & $\mathrm{H}(7)$ & $2.69(7)$ & (1) & $C(2)$ & $\mathrm{H}(8)$ & $3.3(1)$ & (1) \\
\hline$C(2)$ & $\mathrm{H}(2)$ & $2.17(6)$ & (1) & $C(2)$ & $\mathrm{H}(4)$ & $2.89(6)$ & $(1,1,0,-1)$ \\
\hline$C(2)$ & $\mathrm{H}(4)$ & $3.20(6)$ & (1) & $C(2)$ & $\mathrm{H}(5)$ & $2.64(5)$ & (1) \\
\hline$C(2)$ & $\mathrm{H}(6)$ & $3.26(7)$ & (1) & $C(2)$ & $\mathrm{H}(10)$ & $3.5(1)$ & $(2,2,-1,-1)$ \\
\hline$C(2)$ & $\mathrm{H}(9)$ & $2.78(6)$ & (1) & $C(2)$ & $\mathrm{H}(1)$ & $0.95(2)$ & (1) \\
\hline $\mathrm{C}(7)$ & $\mathrm{H}(14)$ & $3.49(8)$ & $(1,1,0,1)$ & $C(7)$ & $\mathrm{H}(2)$ & $2.15(4)$ & (1) \\
\hline$C(7)$ & $\mathrm{H}(4)$ & $2.16(5)$ & (1) & $C(7)$ & $\mathrm{H}(18)$ & $3.5(3)$ & $(2,2,-1,0,-1)$ \\
\hline $\mathrm{C}(7)$ & $\mathrm{H}(19)$ & $3.1(3)$ & $(2,2,-1,0,-1)$ & $C(7)$ & $\mathrm{H}(5)$ & $0.95(2)$ & (1) \\
\hline$C(7)$ & $\mathrm{H}(6)$ & $0.95(2)$ & (1) & $C(7)$ & $\mathrm{H}(11)$ & $3.2(2)$ & $(2,2,-1,-1)$ \\
\hline $\mathrm{C}(7)$ & $\mathrm{H}(9)$ & $3.31(7)$ & (1) & $C(7)$ & $\mathrm{H}(3)$ & $3.08(5)$ & (1) \\
\hline$C(7)$ & $\mathrm{H}(1)$ & $3.12(6)$ & (1) & $\mathrm{C}(11)$ & $\mathrm{H}(18)$ & $3.3(2)$ & $(1,1,0,0,1)$ \\
\hline$C(11)$ & $\mathrm{H}(12)$ & $3.25(9)$ & (1) & $\mathrm{C}(11)$ & $\mathrm{H}(11)$ & $2.03(4)$ & (1) \\
\hline$C(11)$ & $\mathrm{H}(17)$ & $3.2(3)$ & $(1,1,0,0,1)$ & $\mathrm{C}(11)$ & $\mathrm{H}(10)$ & $0.95(2)$ & (1) \\
\hline$C(11)$ & $\mathrm{H}(10)$ & $3.47(2)$ & $(2,2,-1)$ & $\mathrm{C}(11)$ & $\mathrm{H}(9)$ & $2.02(4)$ & (1) \\
\hline$C(11)$ & $\mathrm{H}(9)$ & $3.17(4)$ & $(2,2,-1)$ & $C(9)$ & $\mathrm{H}(7)$ & $2.03(3)$ & (1) \\
\hline$C(9)$ & $\mathrm{H}(8)$ & $2.04(3)$ & (1) & $C(9)$ & $\mathrm{H}(4)$ & $3.21(7)$ & (1) \\
\hline$C(9)$ & $\mathrm{H}(5)$ & $3.4(1)$ & (1) & $C(9)$ & $\mathrm{H}(13)$ & $2.04(4)$ & (1) \\
\hline$C(9)$ & $\mathrm{H}(12)$ & $3.44(6)$ & $(1,1,0,-1)$ & $C(9)$ & $\mathrm{H}(12)$ & $3.29(6)$ & (1) \\
\hline$C(9)$ & $\mathrm{H}(17)$ & $3.4(2)$ & $(1,1,0,0,1)$ & $C(9)$ & $\mathrm{H}(10)$ & $3.29(7)$ & (1) \\
\hline$C(9)$ & $\mathrm{H}(9)$ & $2.06(4)$ & (1) & $C(9)$ & $\mathrm{H}(1)$ & $3.50(6)$ & (1) \\
\hline$C(9)$ & $\mathrm{H}(1)$ & $3.26(6)$ & $(1,1,0,1)$ & $\mathrm{C}(14)$ & $\mathrm{H}(7)$ & $3.06(5)$ & (1) \\
\hline$C(14)$ & $\mathrm{H}(7)$ & $3.14(5)$ & $(1,1,0,1)$ & $\mathrm{C}(14)$ & $\mathrm{H}(8)$ & $2.59(5)$ & (1) \\
\hline
\end{tabular}


Table 9. Distances beyond the asymmetric unit out to $3.60 \approx$ (continued)

\begin{tabular}{|c|c|c|c|c|c|c|c|}
\hline atom & atom & distance & ADC & atom & atom & distance & $\mathrm{ADC}$ \\
\hline$C(14)$ & $\mathrm{H}(13)$ & $0.95(2)$ & (1) & $C(14)$ & $\mathrm{H}(12)$ & $2.05(4)$ & (1) \\
\hline$C(14)$ & $\mathrm{H}(11)$ & $3.26(6)$ & (1) & $C(14)$ & $\mathrm{H}(17)$ & $3.2(3)$ & $(1,1,0,0,1)$ \\
\hline$C(14)$ & $\mathrm{H}(9)$ & $3.26(8)$ & (1) & $C(14)$ & $\mathrm{H}(1)^{\prime}$ & $2.95(9)$ & $(1,1,0,1)$ \\
\hline$C(16)$ & $\mathrm{H}(15)$ & $2.06(5)$ & (1) & $C(16)$ & $\mathrm{H}(14)$ & 2.05(5) & (1) \\
\hline$C(16)$ & $\mathrm{H}(2)$ & $2.98(7)$ & (1) & $C(16)$ & $\mathrm{H}(19)$ & $3.3(1)$ & (1) \\
\hline$C(16)$ & $\mathrm{H}(6)$ & $3.37(7)$ & (1) & $C(16)$ & $\mathrm{H}(20)$ & $2.04(4)$ & (1) \\
\hline$C(16)$ & $\mathrm{H}(16)$ & $2.01(5)$ & (1) & $C(16)$ & $\mathrm{H}(17)$ & $3.26(7)$ & (1) \\
\hline$C(13)$ & $\mathrm{H}(7)$ & $3.13(8)$ & $(1,1,0,1)$ & $C(13)$ & $\mathrm{H}(13)$ & 2.04(4) & (1) \\
\hline$C(13)$ & $\mathrm{H}(12)$ & $0.95(2)$ & (1) & $C(13)$ & $\mathrm{H}(11)$ & $2.03(4)$ & (1) \\
\hline$C(13)$ & $\mathrm{H}(17)$ & $2.9(2)$ & $(1,1,0,0,1)$ & $C(13)$ & $\mathrm{H}(10)$ & 3.2(1) & (1) \\
\hline$C(13)$ & $\mathrm{H}(9)$ & $3.23(7)$ & $(1,1,0,1)$ & $C(13)$ & $\mathrm{H}(1)$ & 3.1(1) & $(1,1,0,1)$ \\
\hline$C(21)$ & $\mathrm{H}(15)$ & $3.2(1)$ & (1) & $C(21)$ & $\mathrm{H}(14)$ & $2.57(9)$ & (1) \\
\hline$C(21)$ & $\mathrm{H}(2)$ & $3.01(5)$ & (1) & $C(21)$ & $\mathrm{H}(18)$ & $3.3(1)^{\prime}$ & (1) \\
\hline$C(21)$ & $\mathrm{H}(18)$ & $3.4(1)$ & $(2,2,-1,-1,-1)$ & $C(21)$ & $\mathrm{H}(19)$ & $2.05(6)$ & (1) \\
\hline$C(21)$ & $\mathrm{H}(20)$ & $0.95(2)$ & (1) & $C(21)$ & $\mathrm{H}(16)$ & $3.23(6)$ & (1) \\
\hline$C(21)$ & $\mathrm{H}(17)$ & $3.20(7)$ & $(1,1,0,-1)$ & $C(20)$ & $\mathrm{H}(18)$ & 2.04(4) & (1) \\
\hline$C(20)$ & $\mathrm{H}(19)$ & $0.95(2)$ & (1) & $C(20)$ & $\mathrm{H}(19)$ & $3.36(2)$ & $(2,2,-1,0,-1)$ \\
\hline$C(20)$ & $\mathrm{H}(6)$ & $3.3(1)$ & $(2,2,-1,-1,-1)$ & $C(20)$ & $\mathrm{H}(12)$ & $3.3(4)$ & $(1,1,0,-1,-1)$ \\
\hline$C(20)$ & $\mathrm{H}(11)$ & $3.0(2)$ & $(1,1,0,-1,-1)$ & $C(20)$ & $\mathrm{H}(20)$ & $2.03(5)$ & (1) \\
\hline$C(20)$ & $\mathrm{H}(20)$ & $3.15(5)$ & $(2,2,-1,0,-1)$ & $C(20)$ & $\mathrm{H}(17)$ & $3.48(8)$ & $(1,1,0,-1)$ \\
\hline$C(20)$ & $\mathrm{H}(17)$ & $3.28(8)$ & (1) & $C(19)$ & $\mathrm{H}(2)$ & $3.5(1)$ & $(2,2,-1,0,-1)$ \\
\hline$C(19)$ & $\mathrm{H}(18)$ & $0.95(2)$ & (1) & $C(19)$ & $\mathrm{H}(19)$ & $2.04(5)$ & (1) \\
\hline$C(19)$ & $\mathrm{H}(19)$ & $3.30(5)$ & $(2,2,-1,0,-1)$ & $C(19)$ & $\mathrm{H}(6)$ & $3.5(1)$ & $(2,2,-1,-1,-1)$ \\
\hline$C(19)$ & $\mathrm{H}(12)$ & $3.0(4)$ & $(1,1,0,-1,-1)$ & $C(19)$ & $\mathrm{H}(11)$ & $3.0(2)$ & $(1,1,0,-1,-1)$ \\
\hline$C(19)$ & $\mathrm{H}(20)$ & $3.26(7)$ & (1) & C(19) & $\mathrm{H}(20)$ & $3.13(7)$ & $(2,2,-1,0,-1)$ \\
\hline$C(19)$ & $\mathrm{H}(16)$ & 3.3(1) & (1) & $C(19)$ & $\mathrm{H}(17)$ & 2.06(4) & (1) \\
\hline$C(6)$ & $\mathrm{H}(7)$ & $3.44(6)$ & (1) & $C(6)$ & $\mathrm{H}(8)$ & 2.88(8) & (1) \\
\hline$C(6)$ & $\mathrm{H}(14)$ & $3.29(7)$ & $(1,1,0,1)$ & $C(6)$ & $\mathrm{H}(2)$ & $3.17(7)$ & (1) \\
\hline$C(6)$ & $\mathrm{H}(4)$ & $0.95(2)$ & (1) & $C(6)$ & $\mathrm{H}(5)$ & $2.12(4)$ & (1) \\
\hline$C(6)$ & $\mathrm{H}(6)$ & $2.12(6)$ & (1) & $C(6)$ & $\mathrm{H}(9)$ & $3.6(1)$ & (1) \\
\hline$C(6)$ & $\mathrm{H}(3)$ & $2.19(5)$ & (1) & $C(6)$ & $\mathrm{H}(1)$ & $3.22(6)$ & (1) \\
\hline$C(6)$ & $\mathrm{H}(1)$ & $3.49(6)$ & $(1,1,0,1)$ & $C(10)$ & $\mathrm{H}(7)$ & $2.87(6)$ & (1) \\
\hline$C(10)$ & $\mathrm{H}(8)$ & $3.31(6)$ & (1) & $C(10)$ & $\mathrm{H}(5)$ & $2.96(5)$ & (1) \\
\hline$C(10)$ & $\mathrm{H}(13)$ & 3.3(1) & (1) & $C(10)$ & $\mathrm{H}(12)$ & $3.27(7)$ & $(1,1,0,-1)$ \\
\hline$C(10)$ & $\mathrm{H}(11)$ & $3.25(7)$ & (1) & $C(10)$ & $\mathrm{H}(17)$ & $3.4(2)$ & $(1,1,0,0,1)$ \\
\hline$C(10)$ & $\mathrm{H}(10)$ & $2.02(3)$ & (1) & $C(10)$ & $\mathrm{H}(10)$ & $3.39(3)$ & $(2,2,-1,-1)$ \\
\hline$C(10)$ & $\mathrm{H}(9)$ & $0.95(2)$ & (1) & $C(10)$ & $\mathrm{H}(1)$ & $3.28(6)$ & (1) \\
\hline$C(5)$ & $\mathrm{H}(15)$ & $2.75(4)$ & (1) & $C(5)$ & $\mathrm{H}(14)$ & $3.29(6)$ & (1) \\
\hline$C(5)$ & $\mathrm{H}(14)$ & $3.31(6)$ & $(1,1,0,1)$ & $C(5)$ & $\mathrm{H}(2)$ & $3.20(6)$ & (1) \\
\hline$C(5)$ & $\mathrm{H}(4)$ & $2.13(4)$ & (1) & $C(5)$ & $\mathrm{H}(5)$ & $3.23(6)$ & (1) \\
\hline $\mathrm{C}(5)$ & $\mathrm{H}(6)$ & $2.61(6)$ & (1) & $C(5)$ & $\mathrm{H}(16)$ & $3.32(8)$ & (1) \\
\hline$C(5)$ & $\mathrm{H}(3)$ & $0.95(2)$ & (1) & $C(15)$ & $\mathrm{H}(15)$ & $0.95(2)$ & (1) \\
\hline
\end{tabular}


Table 9. Distances beyond the asymmetric unit out to $3.60 \AA$ (continued)

$\begin{array}{llllllll}\text { atom } & \text { atom } & \text { distance } & \mathrm{ADC} & \text { atom } & \text { atom } & \text { distance } & \text { ADC } \\ \mathrm{C}(15) & \mathrm{H}(14) & 0.95(2) & (1) & \mathrm{C}(15) & \mathrm{H}(2) & 2.80(7) & (1) \\ \mathrm{C}(15) & \mathrm{H}(4) & 3.58(7) & (1,1,0,-1) & \mathrm{C}(15) & \mathrm{H}(6) & 3.59(7) & (1) \\ \mathrm{C}(15) & \mathrm{H}(20) & 2.67(7) & (1) & \mathrm{C}(15) & \mathrm{H}(16) & 2.68(5) & (1) \\ \mathrm{C}(15) & \mathrm{H}(3) & 2.89(6) & (1) & \mathrm{C}(8) & \mathrm{H}(7) & 0.95(2) & (1) \\ \mathrm{C}(8) & \mathrm{H}(8) & 0.95(1) & (1) & \mathrm{C}(8) & \mathrm{H}(4) & 2.84(5) & (1) \\ \mathrm{C}(8) & \mathrm{H}(5) & 3.6(2) & (1) & \mathrm{C}(8) & \mathrm{H}(13) & 2.69(5) & (1) \\ \mathrm{C}(8) & \mathrm{H}(12) & 3.5(1) & (1,1,0,-1) & \mathrm{C}(8) & \mathrm{H}(9) & 2.72(5) & (1) \\ \mathrm{C}(8) & \mathrm{H}(1) & 2.84(6) & (1) & \mathrm{C}(18) & \mathrm{H}(18) & 2.06(6) & (1) \\ \mathrm{C}(18) & \mathrm{H}(19) & 3.27(7) & (1) & \mathrm{C}(18) & \mathrm{H}(19) & 3.56(7) & (2,2,-1,0,-1) \\ \mathrm{C}(18) & \mathrm{H}(12) & 3.0(2) & (1,1,0,-1,-1) & \mathrm{C}(18) & \mathrm{H}(20) & 3.15(7) & (1,1,0,1) \\ \mathrm{C}(18) & \mathrm{H}(16) & 2.02(6) & (1) & \mathrm{C}(18) & \mathrm{H}(17) & 0.95(2) & (1) \\ \mathrm{C}(3) & \mathrm{H}(15) & 3.4(1) & (1) & \mathrm{C}(3) & \mathrm{H}(14) & 2.87(6) & (1) \\ \mathrm{C}(3) & \mathrm{H}(2) & 0.95(2) & (1) & \mathrm{C}(3) & \mathrm{H}(4) & 3.40(6) & (1,1,0,-1) \\ \mathrm{C}(3) & \mathrm{H}(4) & 3.17(6) & (1) & \mathrm{C}(3) & \mathrm{H}(5) & 2.11(4) & (1) \\ \mathrm{C}(3) & \mathrm{H}(6) & 2.11(4) & (1) & \mathrm{C}(3) & \mathrm{H}(20) & 3.52(8) & (1) \\ \mathrm{C}(3) & \mathrm{H}(10) & 3.6(2) & (2,2,-1,-1) & \mathrm{C}(3) & \mathrm{H}(9) & 3.56(9) & (1) \\ \mathrm{C}(3) & \mathrm{H}(3) & 3.21(5) & (1) & \mathrm{C}(3) & \mathrm{H}(1) & 2.23(4) & (1) \\ \mathrm{C}(12) & \mathrm{H}(19) & 3.5(3) & (1,1,0,1,1) & \mathrm{C}(12) & \mathrm{H}(5) & 3.28(9) & (2,2,-1) \\ \mathrm{C}(12) & \mathrm{H}(13) & 3.25(7) & (1) & \mathrm{C}(12) & \mathrm{H}(12) & 2.03(5) & (1) \\ \mathrm{C}(12) & \mathrm{H}(11) & 0.95(2) & (1) & \mathrm{C}(12) & \mathrm{H}(17) & 2.9(3) & (1,1,0,0,1) \\ \mathrm{C}(12) & \mathrm{H}(10) & 2.02(5) & (1) & \mathrm{C}(12) & \mathrm{H}(10) & 3.33(5) & (2,2,-1) \\ \mathrm{C}(12) & \mathrm{H}(9) & 3.25(6) & (1) & \mathrm{C}(12) & \mathrm{H}(9) & 3.36(6) & (1,1,0,1) \\ \mathrm{C}(12) & \mathrm{H}(9) & 3.51(6) & (2,2,-1) & \mathrm{C}(12) & \mathrm{H}(1) & 3.5(1) & (1,1,0,1) \\ \mathrm{C}(17) & \mathrm{H}(15) & 2.72(8) & (1) & \mathrm{C}(17) & \mathrm{H}(14) & 3.29(6) & (1) \\ \mathrm{C}(17) & \mathrm{H}(18) & 3.3(1) & (1) & \mathrm{C}(17) & \mathrm{H}(6) & 3.35(8) & (1) \\ \mathrm{C}(17) & \mathrm{H}(12) & 3.3(2) & (1,1,0,-1,-1) & \mathrm{C}(17) & \mathrm{H}(20) & 3.24(6) & (1) \\ \mathrm{C}(17) & \mathrm{H}(20) & 3.42(6) & (1,1,0,1) & \mathrm{C}(17) & \mathrm{H}(16) & 0.95(2) & (1) \\ \mathrm{C}(17) & \mathrm{H}(17) & 2.03(4) & (1) & \mathrm{C}(17) & \mathrm{H}(3) & 3.6(2) & (1) \\ & & & & & & & \end{array}$

*ADC (S, L, TX, TY, TZ) represents:

S: Symmetry operation number. $-S$ is inversion of operation $S$.
( 1) $X, Y, Z$
( 2) $-X, Y,-Z$

L: Lattice translation.

( 1) $0,0,0$

( 2) $1 / 2,1 / 2,0$

TX, TY, TZ: Unit cell translation along the $\mathrm{x}, \mathrm{y}$, and $\mathrm{z}$ directions. 